\title{
Recent Development of Non-Contact Multi-Target Vital Sign Detection and Location Tracking Based on Metamaterial Leaky Wave Antennas
}

\author{
Yichao Yuan and Chung-Tse Michael Wu *(D) \\ Department of Electrical \& Computer Engineering, Rutgers University, Piscataway, NJ 08854, USA; \\ yichao.yuan@rutgers.edu \\ * Correspondence: ctm.wu@rutgers.edu; Tel.: +1-848-445-5393
}

Citation: Yuan, Y.; Wu, C.-T.M

Recent Development of Non-Contact Multi-Target Vital Sign Detection and Location Tracking Based on Metamaterial Leaky Wave Antennas. Sensors 2021, 21, 3619. https:// doi.org/10.3390/s21113619

Academic Editor:

Theodore E. Matikas

Received: 16 April 2021

Accepted: 19 May 2021

Published: 22 May 2021

Publisher's Note: MDPI stays neutra with regard to jurisdictional claims in published maps and institutional affiliations.

Copyright: (c) 2021 by the authors. Licensee MDPI, Basel, Switzerland. This article is an open access article distributed under the terms and conditions of the Creative Commons Attribution (CC BY) license (https:/ / creativecommons.org/licenses/by/ $4.0 /)$.

\begin{abstract}
Microwave radar sensors have been developed for non-contact monitoring of the health condition and location of targets, which will cause minimal discomfort and eliminate sanitation issues, especially in a pandemic situation. To this end, several radar sensor architectures and algorithms have been proposed to detect multiple targets at different locations. Traditionally, beamforming techniques incorporating phase shifters or mechanical rotors are utilized, which is relatively complex and costly. On the other hand, metamaterial (MTM) leaky wave antennas (LWAs) have a unique property of launching waves of different spectral components in different directions. This feature can be utilized to detect multiple targets at different locations to obtain their healthcare and location information accurately, without complex structure and high cost. To this end, this paper reviews the recent development of MTM LWA-based radar sensor architectures for vital sign detection and location tracking. The experimental results demonstrate the effectiveness of MTM vital sign radar compared with different radar sensor architectures.
\end{abstract}

Keywords: metamaterial (MTM); leaky wave antenna (LWA); radar sensor architecture; vital sign detection; location tracking

\section{Introduction}

Microwave radar sensors have been developed in physiological [1-10] or location tracking [11-16] of human targets over the past decades, with the development of semiconductors and algorithms. Owing to non-contact and penetrable characteristics of the radar sensor, this will cause minimal discomfort for the detected target and avoid the sanitation issues, which has gained much interest in the pandemic situation. Among them, the conventional homodyne radar sensor for vital sign detection illuminates the transmitted radiofrequency (RF) signal towards the target via the transmitting (Tx) antenna, while the reflected signal received by the receiving $(\mathrm{Rx})$ antenna will be down-converted to the baseband signal through the mixer, as shown in Figure 1. Efforts have been made by researchers to improve the homodyne radar sensor architecture in recent years, in order to achieve accurate performance on vital sign detection. For example, quadrature Doppler radar receiver system is employed to solve the null point problem [17]. Furthermore, to mitigate the strong interference signal that is mainly caused by random body movement, a two-transceiver radar system with different polarizations and frequencies is placed at different body orientations to detect vital sign signals [18]. Another challenge for vital sign detection based on this architecture is $1 / \mathrm{f}$ noise, which can be overcome by low intermediate-frequency (IF) heterodyne radar sensor architectures [19].

Besides vital sign detection, microwave radar sensors can also be used for location tracking of the target. In [20], the distance to the target can be acquired by analyzing the direct current $(\mathrm{dc})$ information associated with the target's position from baseband signals with different beam-steering angles. On the other hand, a frequency-modulated 
continuous-wave (FMCW) modulation scheme is proposed to detect the distance to the target [21]. While the distance to the target can be obtained, directional information of targets is still a challenge for location tracking. To this end, mechanical rotors [22,23] and phased arrays [24] are proposed to detect different targets at different locations and obtain their directional information, respectively. Nonetheless, mechanical devices are bulky while phased arrays are complex and costly.

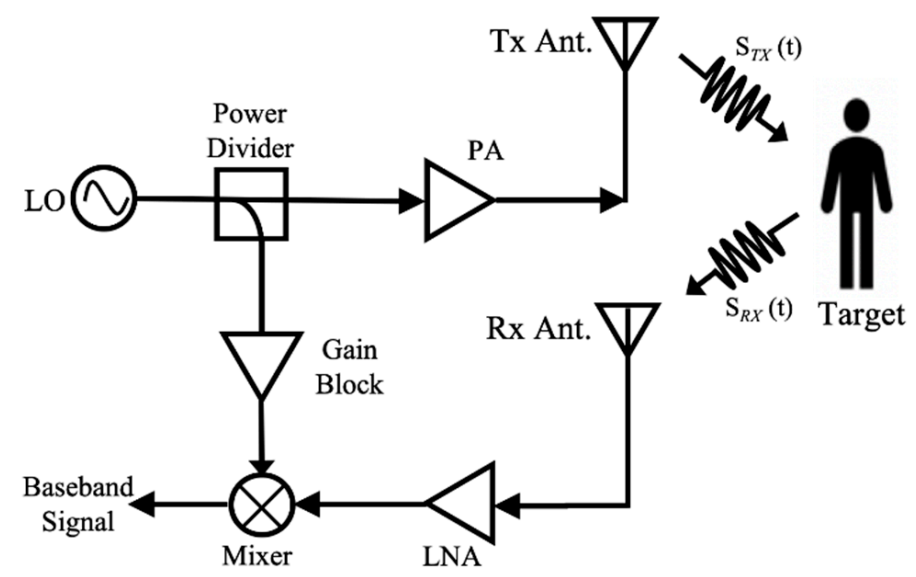

Figure 1. Block diagram of the homodyne radar sensor architecture.

Recently, multiple-input multiple-output (MIMO) radar systems [25-27] have attracted considerable attention because of their capability for measuring angle-of-arrival (AOA) from the received signal, which is an indicator for the estimation of the target's location with directional information. However, the implementation of multiple transceivers increase the systematic complexity and cost. As such, efforts have been made to reduce the systematic complexity. For instance, single-input multiple-output (SIMO) with different mechanisms, such as the digital beam forming (DBF) technique [28] or angle division multiplexing sensing (ADMS) [29], as well as the RF switch-based radar system [30] using a transceiver instead of a multiple radar system are employed to detect multiple targets. Although the number of transceivers can be effectively reduced, the radar systems still adopt multiple antennas at the transmitter or receiver end. Furthermore, the implementation of a control algorithm for the selection of corresponding antennas still increases the complexity of radar system.

On the other hand, metamaterial leaky wave antennas (MTM LWAs) [31] exhibit a unique frequency to space mapping feature, which can be used to overcome the aforementioned shortcomings. In general, MTM is an artificial structure with unusual properties, such as negative index of refraction, which cannot be found in nature. It was first investigated by the Russian physicist Viktor Veselago in 1967, which he termed left-handed (LH) substance [32]. After more than 30 years, inspired by the work of J.B. Pendry et al., LH material was realized by Smith and colleagues at the University of California, San Diego (UCSD), consisting of copper split-ring resonators (SRRs) and thin copper wires, which exhibit negative permittivity $(\varepsilon)$ and permeability $(\mu)$ [33]. In 2002, Caloz et al. [34] and Iyer et al. [35] proposed LH materials based on a transmission line (TL) structure. However, in practice, it is impossible to implement a purely LH TL structure because of unavoidable right-handed (RH) parasitic series inductance and shunt capacitance effects. Therefore, a composite right/left-handed (CRLH) structure is introduced in [31,36,37] to form MTM TLs. It is noted that the CRLH structure can also be realized based on the resonant-type approach [38], such as the use of split ring resonators (SRRs) or complementary split ring resonators (CSRRs). Based on the CRLH structure, MTM LWAs are shown to exhibit frequency-dependent beam-steering capability at the fundamental mode $(n=0)$ [39-42], which is different from other fundamentally slow-wave periodic structure-based LWAs operating at the higher spatial harmonics $(|n|>0)$ in order to generate fast waves, where 
$n$ is an integer representing the order of spatial harmonic [43-45]. Figure 2 illustrates the equivalent circuit of a CRLH TL unit cell, which can exhibit backward-to-forward frequency-dependent beam scanning capability. Such characteristics have been widely used in antenna applications [46-48].

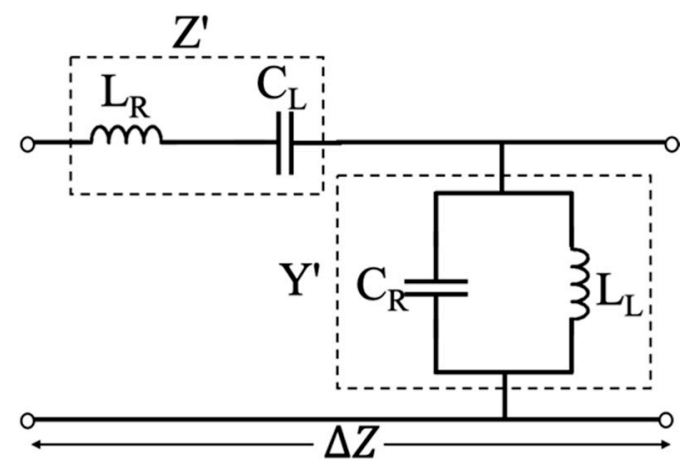

Figure 2. Equivalent Circuit of composite right/left-handed transmission line (CRLH TL) unit cell.

As shown in Figure 2, the CRLH TL unit cell consists of LH series capacitor $\left(C_{L}\right)$ and shunt inductor $\left(L_{L}\right)$ with parasitic RH series inductor $\left(L_{R}\right)$ and shunt capacitor $\left(C_{R}\right)$. Its general propagation constant can be obtained as:

$$
\gamma=j \beta=\frac{1}{\Delta z} \sqrt{Z^{\prime} Y^{\prime}}
$$

where,

$$
Z^{\prime}=j \omega L_{R}+\frac{1}{j \omega C_{L}}, Y^{\prime}=j \omega C_{R}+\frac{1}{j \omega L_{L}},
$$

which leads to:

$$
\beta(\omega)=\frac{s(\omega)}{\Delta z} \sqrt{\omega^{2} L_{R} C_{R}+\frac{1}{\omega^{2} L_{L} C_{L}}-\left(\frac{L_{R}}{L_{L}}+\frac{C_{R}}{C_{L}}\right)},
$$

where $\beta(\omega)$ is the phase constant of a TL, s( $\omega)$ and $\Delta z$ are the sign function and the length of unit cell, respectively. For the balanced case, i.e., $L_{R} C_{L}=L_{L} C_{R}$, Equation (3) can be simplified as:

$$
\beta(\omega)=\beta_{R H}+\beta_{L H}=\frac{\omega}{\Delta z} \sqrt{L_{R} C_{R}}-\frac{1}{\omega \Delta z \sqrt{L_{L} C_{L}}},
$$

Equation (4) shows the phase constant $\beta(\omega)$ can be changed from negative value to positive value as the frequency increases, which corresponds to the left-handed (LH) effects and right-handed (RH) effects. Its dispersion diagram is shown in Figure 3. In the fast wave region where the phase velocity is greater than the speed of light, radiation occurs with frequency-dependent radiation main beam angle $\theta$ for a CRLH TL given by Figure 4 .

Specifically, the radiation angle of the main beam can be expressed as:

$$
\theta(\omega)=\sin ^{-1}\left(\frac{\beta(\omega)}{k_{0}}\right)
$$

where $k_{0}$ is the free space wave number. As the frequency varies, phase constant $\beta(\omega)$ of the CRLH TL is changed, which leads to the change of $\theta(\omega)$. As a result, the CRLH MTM LWA is able to scan from the backfire to endfire direction, when $\beta(\omega)$ changes from negative to positive according to Equation (5). Owing to the frequency-dependent beam scanning capability of the MTM LWA, the target directional information can be easily found based on its radiation pattern and operational frequency. 


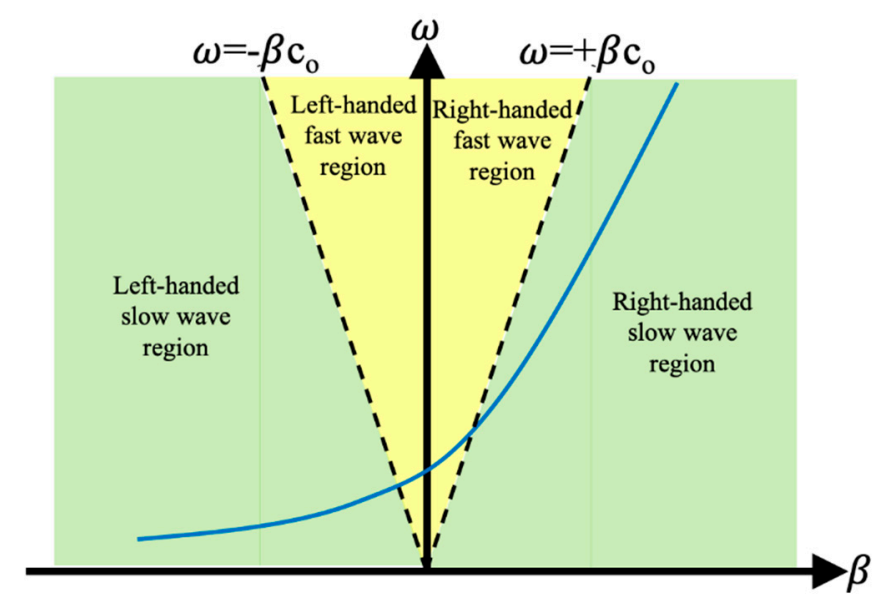

Figure 3. Dispersion diagram of CRLH TL (balanced case).

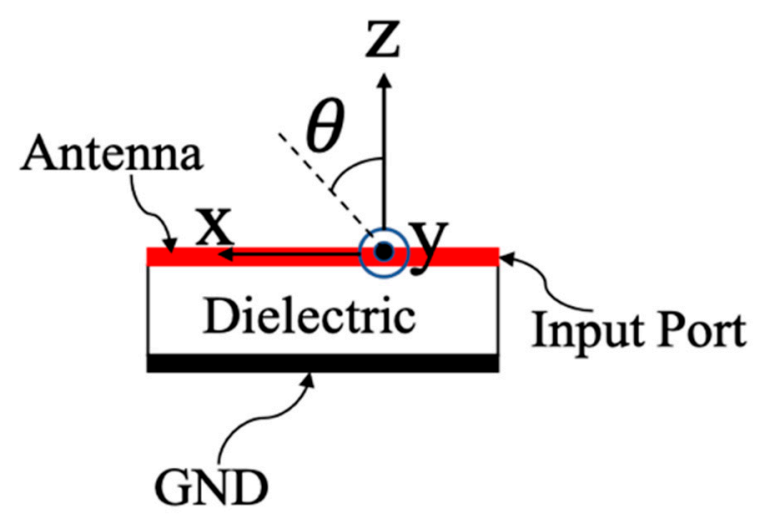

Figure 4. The radiation angle of CRLH TL.

The rest of this paper is organized as follows. Section 2 reviews homodyne architectures with one-dimensional (1D) MTM LWAs [49,50], which are employed for vital sign detection and location tracking based on the arctangent demodulation. Section 3 presents a self-injection-locked (SIL) radar architecture integrated with MTM LWAs to detect vital sign signals and track locations using frequency-shift keying (FSK) method [51]. Section 4 introduces a two-dimensional (2D) MTM LWA array for vital sign detection at $24 \mathrm{GHz}$ band [52]. Finally, conclusions are made in Section 5.

\section{Metamaterial Leaky Wave Antenna (MTM LWA)-Based Homodyne Architecture}

While conventional microwave radars have been widely studied for vital sign detection and location tracking of single target [17,53-55], the ability to perform multi-target detection has gained much interest recently [56,57]. Lu et al. applied a linearly polarized MTM LWA structure at $24 \mathrm{GHz}$ band to detect the vital sign information of multiple targets and track their motions with homodyne architecture [49]. Figure 5 shows the layout and the radiation pattern of the 1D frequency-dependent beam scanning MTM LWA. In this design, a 1D frequency-dependent beam scanning MTM LWA consists of vialess unit cells with gap capacitors and open stub-loaded resonators, where $C_{L}$ is provided by a gap capacitor and $L_{L}$ is the inductance of an open stub, $L_{R}$ represents that parasitic inductance caused by current gradient flowing across gap capacitor, and $C_{R}$ is provided by the parallel plate effects between the signal layer and ground plane. According to its radiation pattern, the scanning angle of the MTM LWA can be changed from $-30^{\circ}$ to $+50^{\circ}$ as the operation frequency sweeps from $24.3 \mathrm{GHz}$ to $27.3 \mathrm{GHz}$. It is noted that there is a $3 \mathrm{~dB}$ decrease in the radiation gain at the broadside, which may be avoided by forcing both series and shunt elements to contribute equally to the radiation $[58,59]$. 


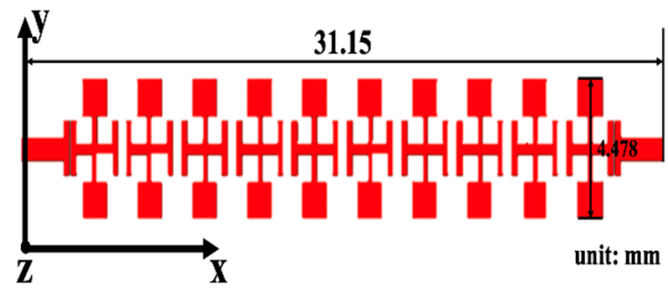

(a)

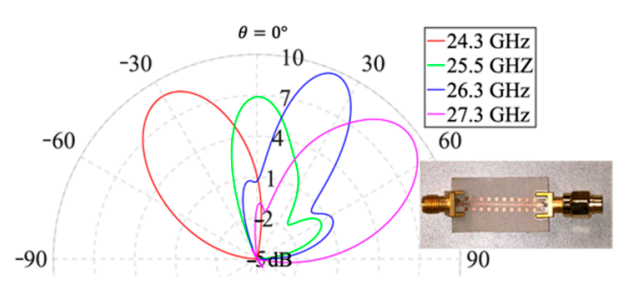

(b)

Figure 5. (a) Layout of 1D metamaterial leaky wave antenna (MTM LWA) at $24 \mathrm{GHz}$ band; (b) its radiation pattern [49].

The system block diagram of the proposed radar sensor module is shown in Figure 6. The radar transceiver chip is employed to control the operation frequency of MTM LWA. Consequently, based on its radiation pattern, the interrogating signal is transmitted via Tx MTM LWA towards the target along a specified direction, which can be expressed as:

$$
\theta(\omega)=\sin ^{-1}\left(\frac{\beta(\omega)}{k_{0}}\right)
$$

where $f$ represents the operation frequency of MTM LWA and $\Phi(t)$ is the phase noise. The reflected signal, modulated by the target, is received by the Rx MTM LWA. Then it is down-converted to the I- and Q-baseband signals. A micro controller unit (MCU) digitalizes the signals, which are then transmitted through Bluetooth to the laptop for post processing. By normalizing the signal amplitude, the digitized I- and Q-baseband signals can be expressed as:

$$
\begin{aligned}
& B_{I}(n)=\cos \left[\frac{4 \pi d_{0}}{\lambda}+\frac{4 \pi x_{v}(n)}{\lambda}+\theta_{0}+\Delta \Phi\right]+D C_{I}(n), \\
& B_{Q}(n)=\sin \left[\frac{4 \pi d_{0}}{\lambda}+\frac{4 \pi x_{v}(n)}{\lambda}+\theta_{0}+\Delta \Phi\right]+D C_{Q}(n),
\end{aligned}
$$

where $d_{0}$ is the nominal distance from the radar sensor to the target, $\theta_{0}$ accounts for the phase response due to the phase change at the target surface and phase delay between the antenna and mixer, $\Delta \Phi$ represents the phase residue, and $D C_{I}(n) / D C_{Q}(n)$ are the dc offset of the in-phase and quadrature $(\mathrm{I} / \mathrm{Q})$ channels. Because $x_{v}(n)$ represents the displacement related to the vital sign signal of target, it can be denoted as:

$$
x_{v}(n)=A_{b r} \cos \left(2 \pi f_{b r} t\right)+B_{h} \cos \left(2 \pi f_{h} t\right),
$$

where $A_{b r}, B_{h}$ are the displacements related to respiration and heartbeat activities and $f_{b r}$, $f_{h}$ are their corresponding frequencies. After calibrating the dc offset, the vital sign signals can be extracted in the frequency domain by performing fast Fourier transform (FFT) based on the arctangent demodulation method.

In the experimental setup, shown in Figure 7, the vital sign signals of two targets sitting in front of the radar at different directions are chosen to be detected using the proposed radar sensor module. Target A sits at $\theta=-30^{\circ}$ in the backward side and target B sits at $\theta=+30^{\circ}$ in the forward side, in which the corresponding frequencies are $24.3 \mathrm{GHz}$ and $26.3 \mathrm{GHz}$, according to the radiation patterns of MTM LWA. The distance to the targets is $0.8 \mathrm{~m}$.

For vital sign information of target $\mathrm{A}$, the I- and Q-baseband signals in the time domain are shown in Figure 8a. As a result, corresponding respiration and heartbeat rate in the frequency domain are shown in Figure 8b. In the same way, the I- and Q-baseband signals for the vital sign information signal of Target $\mathrm{B}$ and his corresponding respiration and heartbeat rate are shown in Figure 9. 


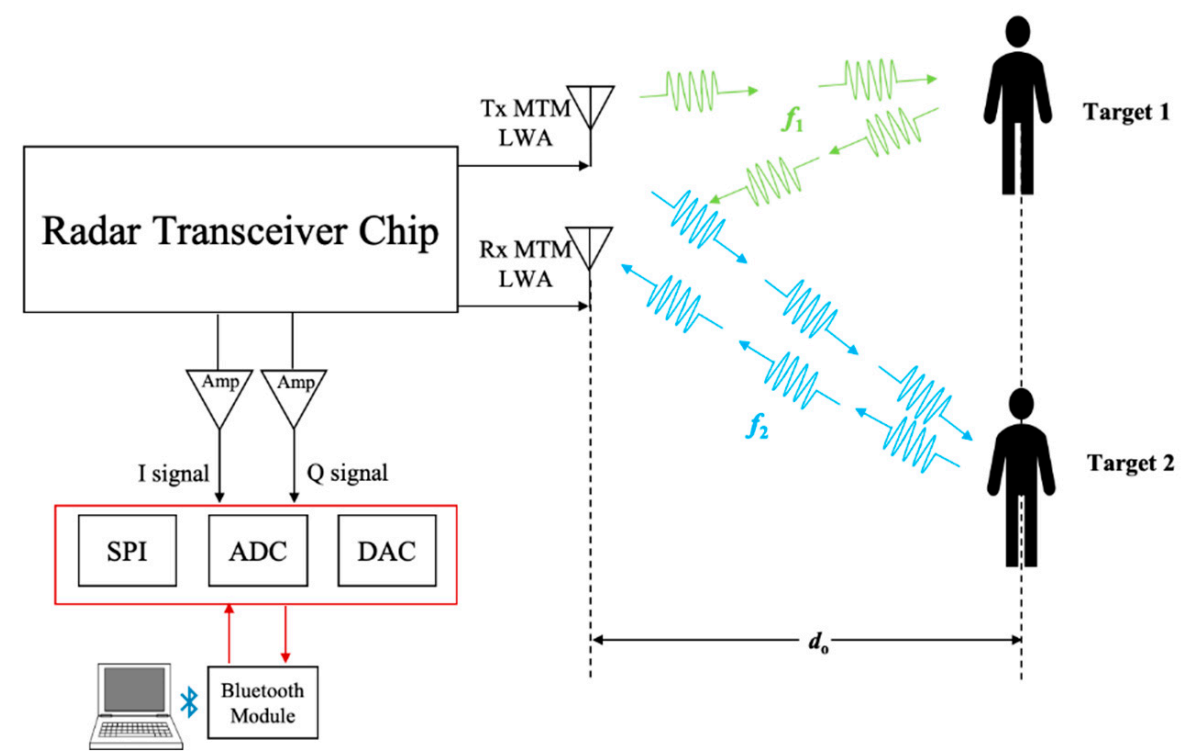

Figure 6. The block diagram of MTM LWA-based homodyne architecture at $24 \mathrm{GHz}$ band.

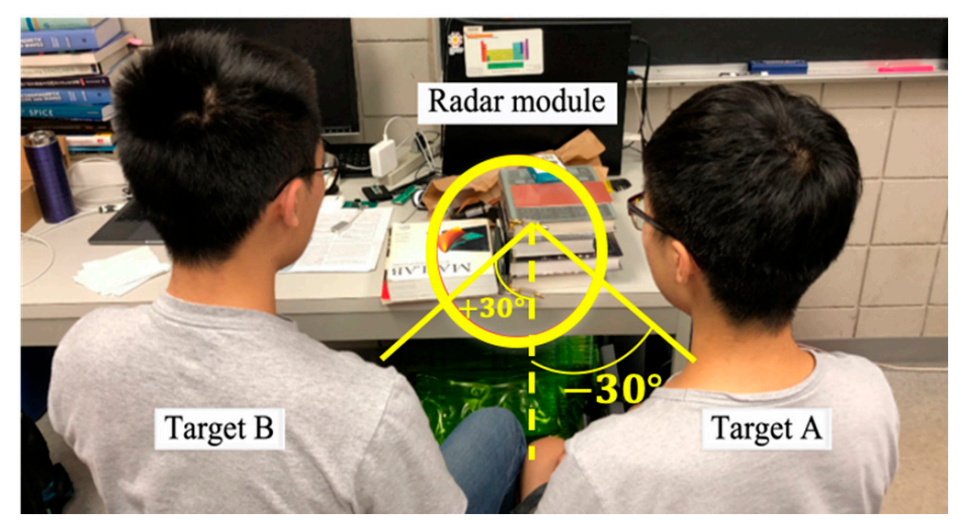

Figure 7. Experimental setup for detecting vital sign signals of two targets at different locations using MTM LWA-based homodyne radar sensor module at $24 \mathrm{GHz}$ band [49].

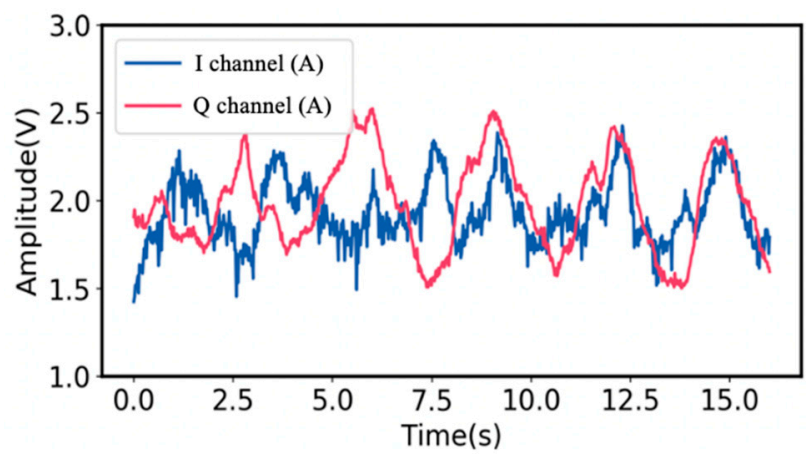

(a)

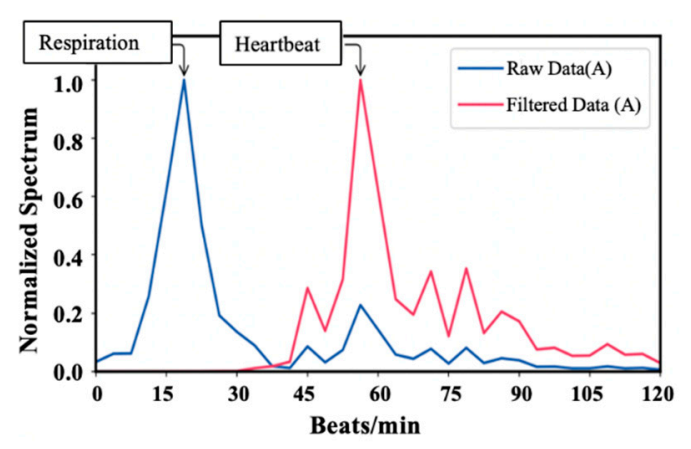

(b)

Figure 8. (a) Time domain vital sign signal of Target A; (b) the measured respiration and heartbeat rates of Target A using MTM LWA-based homodyne radar sensor module band [49]. 


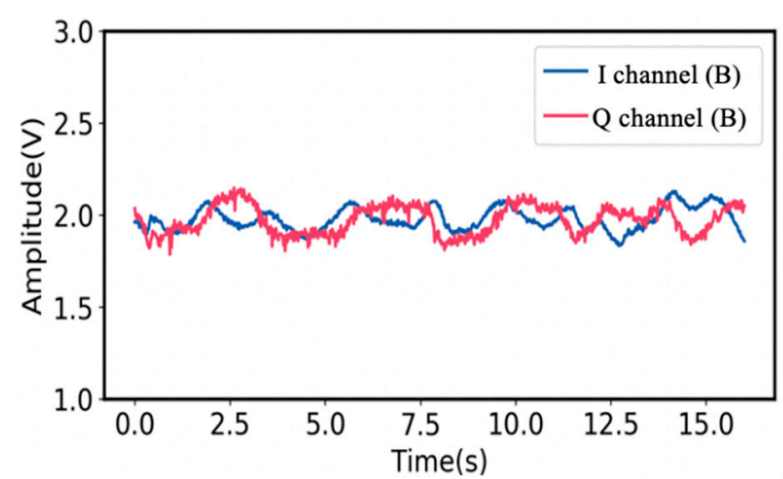

(a)

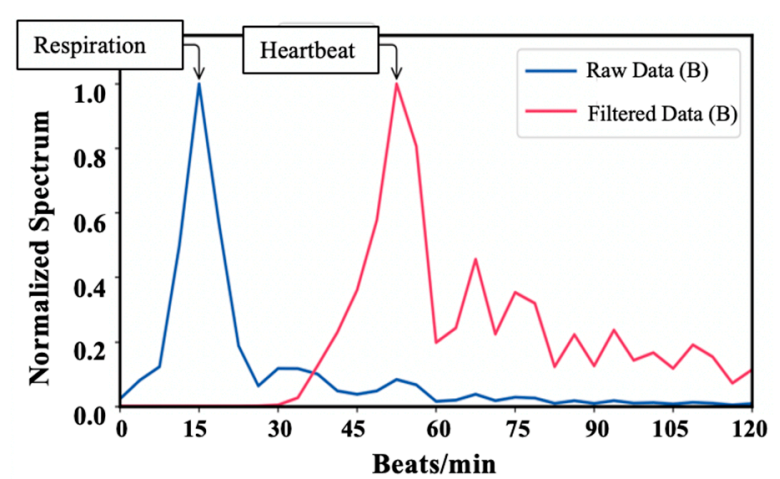

(b)

Figure 9. (a) Time domain vital sign signal of Target B; (b) the measured respiration and heartbeat rates of Target B using MTM LWA-based homodyne radar sensor module band [49].

It is worth mentioning that a digital Butterworth high pass filter with the cutoff frequency of $0.5 \mathrm{~Hz}$ is employed to identify the heartbeat rate in the frequency domain in case the heartbeat information is overwhelmed by the harmonics coming from the stronger respiration signals. After performing FFT and filtering, the respiration and heartbeat rate of Target A are 18.6 beats $/ \mathrm{min}$ and 56.4 beats $/ \mathrm{min}$, while the ground truth of the respiration and heartbeat rate of Target $\mathrm{A}$ are 17 beats $/ \mathrm{min}$ and 58 beats $/ \mathrm{min}$, respectively. On the other hand, for Target $\mathrm{B}$, the respiration and heartbeat rate are 15 beats $/ \mathrm{min}$ and 56 beats/min, while the ground truth are 15 beats/min and 59 beats/min, respectively.

In addition to the vital sign signal detection, the target motion can also be tracked by using MTM LWA-based homodyne radar sensor module [50]. According to Equations (7) and (8), the I- and Q-baseband signals incorporate distance information between radar sensor and the target. Therefore, by calibrating the dc offset, the I- and Q-baseband signals can be expressed as:

$$
\begin{aligned}
& B_{I}(n)=\cos \left[\frac{4 \pi d_{0}}{\lambda}+\frac{4 \pi x_{v}(n)}{\lambda}+\theta_{0}+\Delta \Phi\right], \\
& B_{Q}(n)=\sin \left[\frac{4 \pi d_{0}}{\lambda}+\frac{4 \pi x_{v}(n)}{\lambda}+\theta_{0}+\Delta \Phi\right],
\end{aligned}
$$

By applying the arctangent demodulation method [17], the phase information of baseband signals can be extracted as:

$$
\Phi(n)=\tan ^{-1}\left(\frac{B_{Q}(n)}{B_{I}(n)}\right)=\frac{4 \pi d_{0}}{\lambda}+\frac{4 \pi x_{v}(n)}{\lambda}+\theta_{0}+\Delta \Phi,
$$

where the small movement $x_{v}(n)$ can be neglected compared with the distance $d_{0}$, and $\Delta \phi$ can be ignored due to the range correlation theory with a small detection displacement [60]. Assuming $\theta_{0}$ is a constant for the target, the relative distance $\Delta d=d_{0}\left(n_{1}\right)-d_{0}\left(n_{2}\right)$ can be obtained based on Equation (13):

$$
\Delta d \approx \frac{\lambda \cdot \Delta \Phi(n)}{4 \pi},
$$

The experimental setup of the multi-target motion detection is shown in Figure 10a. The main beam of the radar sensor illuminates two human targets with different operation frequencies. In this case, Target A moves back and forth with directional angle at $\theta=-30^{\circ}$ in the backward side of the MTM LWA and Target B moves back and forth with directional angle at $\theta=+30^{\circ}$ in the forward side of the MTM LWA. The top view sketch is shown in Figure 10b, where the corresponding operation frequencies of MTM LWA are $24.3 \mathrm{GHz}$ and $26.3 \mathrm{GHz}$, respectively. 


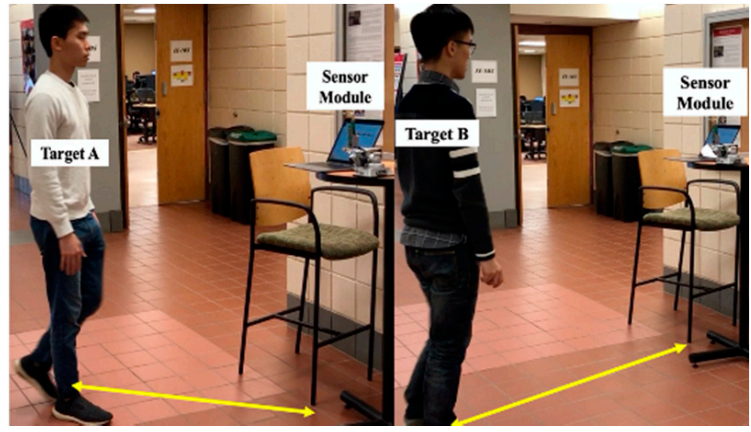

(a)

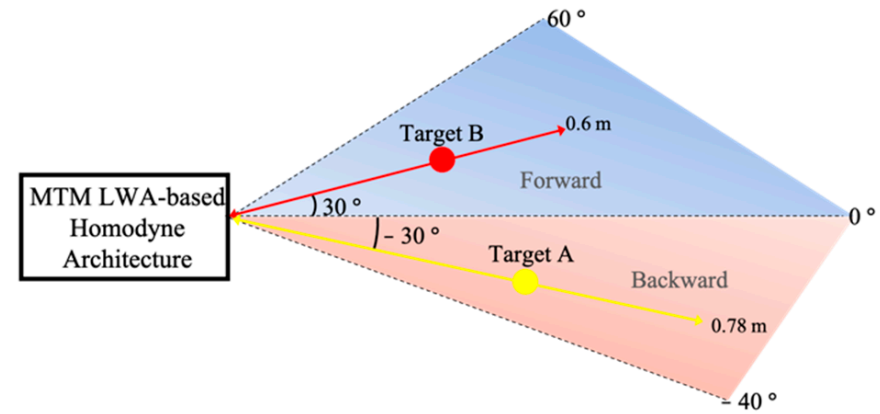

(b)

Figure 10. (a) Experimental setup for multi-target motion detection using MTM LWA-based homodyne radar sensor module; (b) its top view sketch.

The I- and Q-baseband signals for Target A motion and the measured relative moving distance, based on Equation (13), are shown in Figure 11. For Target B, the I- and Qbaseband signals and its measured relative moving distance are plotted in Figure 12. As can be seen, for Target A, the measured relative moving distance is from 0 to $63 \mathrm{~cm}$, while the ground truth is from 0 to $60 \mathrm{~cm}$, leading to $5 \%$ deviation. On the other hand, for Target $\mathrm{B}$, the measured relative moving distance is from 0 to $75 \mathrm{~cm}$ and the ground truth is from 0 to $78 \mathrm{~cm}$, which only leads to $3.8 \%$ deviation.

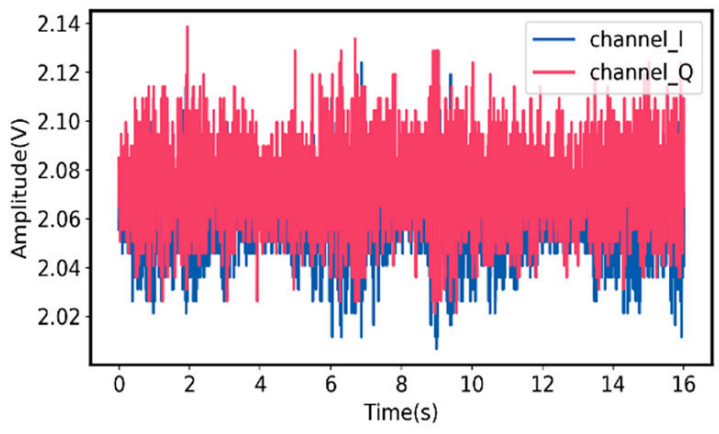

(a)

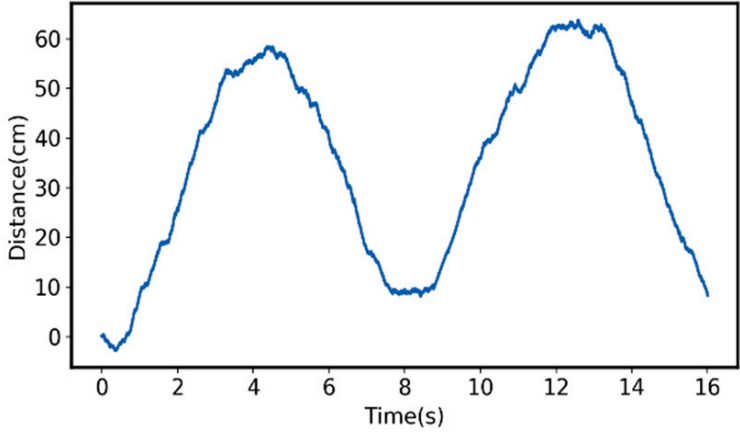

(b)

Figure 11. (a) Measured I- and Q-baseband signals for Target A motion at $24.3 \mathrm{GHz}$; (b) measured relative moving distance of Target A [50].

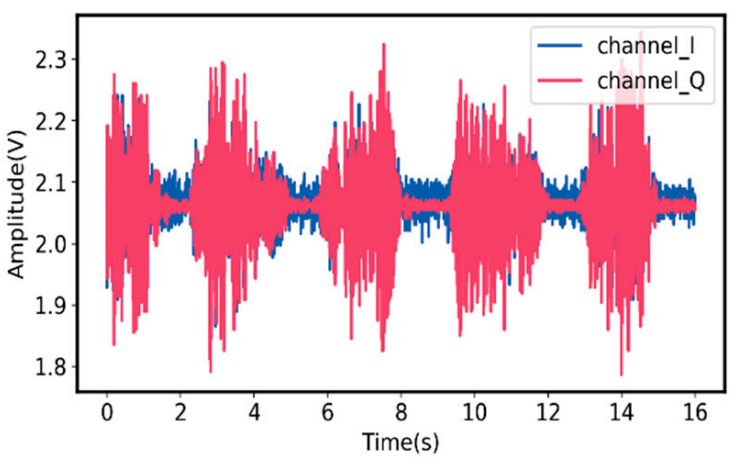

(a)

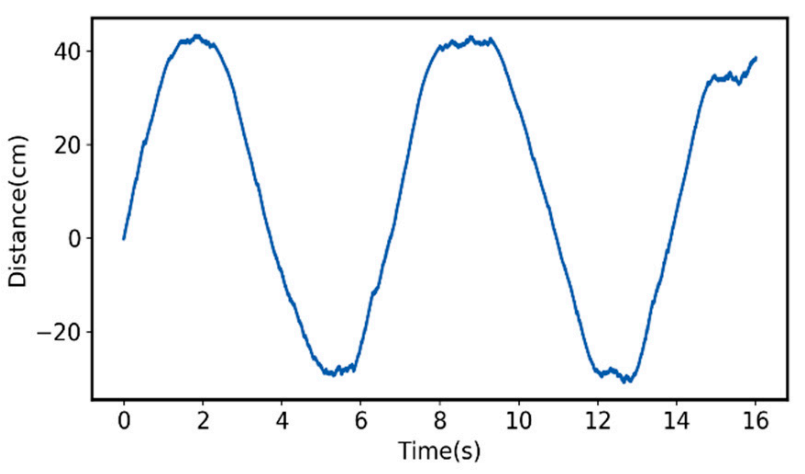

(b)

Figure 12. (a) Measured I- and Q-baseband signals for Target B motion at $26.3 \mathrm{GHz}$; (b) measured relative moving distance of Target B [50]. 


\section{MTM LWA Based on Self-Injection Locked (SIL) Radar Architecture}

In addition to conventional radar sensor architectures, vital sign radar detection based on self-injection locked (SIL) architecture has been proposed recently [61], which has gained much attention because of its low system complexity and high sensitivity [61,62]. Yuan et al. [51] proposed a new architecture of SIL radar sensor integrated with an MTM LWA, where its block diagram is shown in Figure 13. In this scenario, the interrogating RF signal with free-running oscillation frequency, $\omega_{\text {osc }}$, is illuminated via an MTM LWA coming from a self-injection locked oscillator (SILO). The reflected signal modulated by the target locks the oscillator into SIL state. Therefore, the output frequency $\omega_{\text {out }}(t)$ of the SILO will be injection-locked to a different value. Based on Adler's equation [62], it can be expressed as:

$$
\omega_{\text {out }}(t)=\omega_{\text {osc }}-\omega_{\text {LR }} \cos \alpha(t),
$$

where $\omega_{L R}$ denotes as the locking range and $\alpha(t)$ is phase delay of SIL path, which can be given by:

$$
\begin{gathered}
\omega_{L R}=\frac{\omega_{o s c}}{2 Q_{\text {tank }}} \frac{E_{\text {inj }}}{E_{o s c}}, \\
\alpha(t)=\frac{2 \omega_{o s c}}{c}\left(d_{o}+x_{v}(t)\right),
\end{gathered}
$$

where $Q_{\text {tank }}$ is the quality factor of SILO's tank circuit, $E_{i n j}$ is the amplitude of injection signal and $E_{\text {osc }}$ is the amplitude of the free-running signal of the SILO. Therefore, the frequency-modulated output signal of MTM LWA is:

$$
\mathrm{S}_{\text {out }}(t)=A_{\text {out }} \cos \left(\omega_{\text {out }} t\right)
$$

where $A_{\text {out }}$ is the amplitude of output signal. The signal will then be sent into a firstorder microwave differentiator to convert the frequency modulation (FM) of signal to an amplitude modulation (AM) signal, which is given by:

$$
\frac{d \mathrm{~S}_{\text {out }}(t)}{d t}=-\omega_{\text {out }} A_{\text {out }} \sin \left(\omega_{\text {out }} t\right),
$$

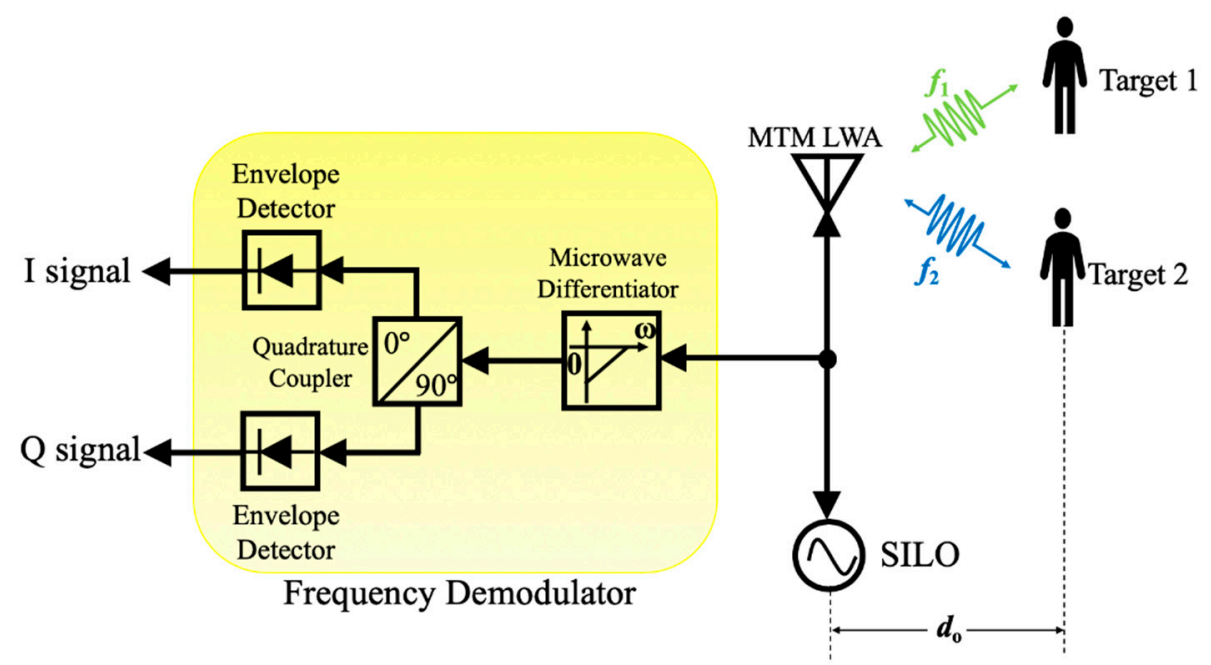

Figure 13. The block diagram of proposed MTM LWA based on self-injection-locked (SIL) radar sensor architecture, illuminating two distinct operation frequencies to two targets.

A quadrature coupler followed by two envelope detectors is then utilized to perform AM demodulation for the I/Q channel. The vital sign signals with distance information of multiple targets at different locations can be obtained by performing FFT of the demodulated I/Q signals. 
To detect multiple targets at different locations, a 1D linearly polarized frequencydependent beam scanning MTM LWA with MTM-based coupler operating at the $2.4 \mathrm{GHz}$ band is designed. Its layout and prototype are shown in Figure 14, which consists of 10 CRLH unit cells. Based on its radiation pattern shown in Figure 15a, the scanning angle of MTM LWA can be changed from $-50^{\circ}$ to $+30^{\circ}$ when the operation frequency varies from $1.85 \mathrm{GHz}$ to $2.85 \mathrm{GHz}$. The reflection coefficient $\left(S_{11}\right)$ is close to $-10 \mathrm{~dB}$ within the same operation frequency range, as shown in Figure 15b.

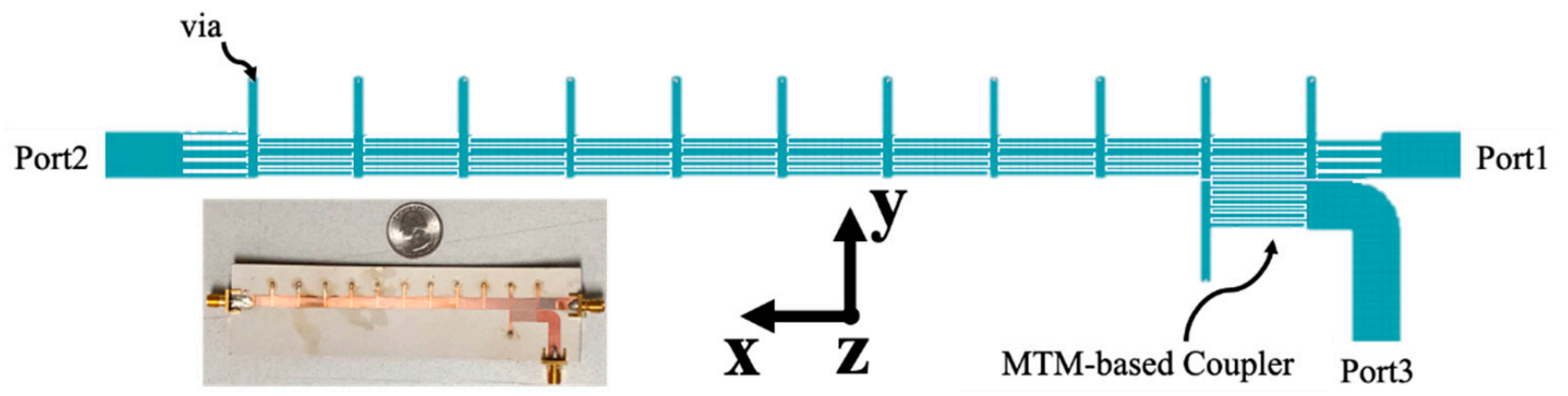

Figure 14. Layout and prototype of 1-D MTM LWA at 2.4 GHz.

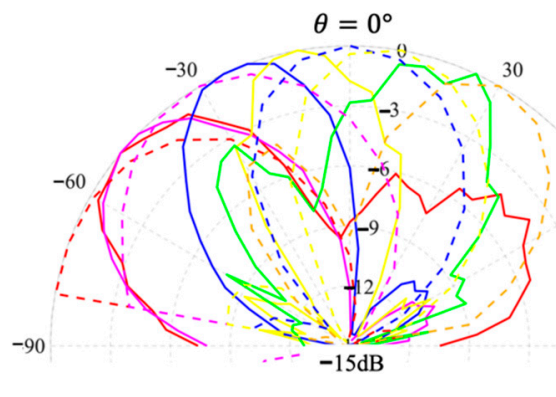

(a)

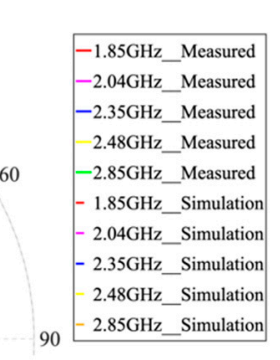

Figure 15. (a) Radiation pattern of 1-D MTM LWA at 2.4 GHz; (b) its S-parameters [51].

Figure 16 presents the prototype of the radar sensor module. The operation frequency of MTM LWA can be tuned from $2.04 \mathrm{GHz}$ to $2.48 \mathrm{GHz}$ through a tunable oscillator, which is designed based on the negative resistance method [63] with common source configuration. The oscillator output is connected to Port 1 of the MTM LWA integrated with the MTM-based coupler to transmit and receive RF signals. When the modulated signal within the frequency band of $2.04 \mathrm{GHz}$ to $2.48 \mathrm{GHz}$ is received by MTM LWA, it will be sent through the MTM-based coupler to the first-order microwave differentiator for frequency demodulation [64], whose magnitude response is linear with respect to the frequency from dc to $3.1 \mathrm{GHz}$ [65]. Consequently, the modulated signal can be converted to the RF signal with the corresponding frequency-dependent amplitudes. Then a quadrature coupler followed by the two envelope detectors is included to demodulate the frequency dependent amplitude signal into I/Q signals for further post processing.

To validate the proposed MTM SIL radar, the experimental setup and its top view sketch are shown in Figure 17. In Figure 17a, Target 1 is located at a distance of $0.6 \mathrm{~m}$ with $\theta=-10^{\circ}$, whereas Target 2 is located at a distance of $0.65 \mathrm{~m}$ with $\theta=-40^{\circ}$ in the backward side. The breath rate and heartbeat incorporated with a high pass filter at the cutoff frequency of $0.5 \mathrm{~Hz}$ for both targets are presented in Figure 18. The measured respiration rates for target 1 and target 2 are 18.3 beats $/ \mathrm{min}$ and $18 \mathrm{beat} / \mathrm{min}$, while the ground truth for both targets is 18 beats $/ \mathrm{min}$. The heartbeat rates for target 1 and 
2 are 85.8 beats $/ \mathrm{min}$ and 81.9 beats/min, while the ground truth are 87 beats $/ \mathrm{min}$ and 81 beats/min for both targets, respectively.
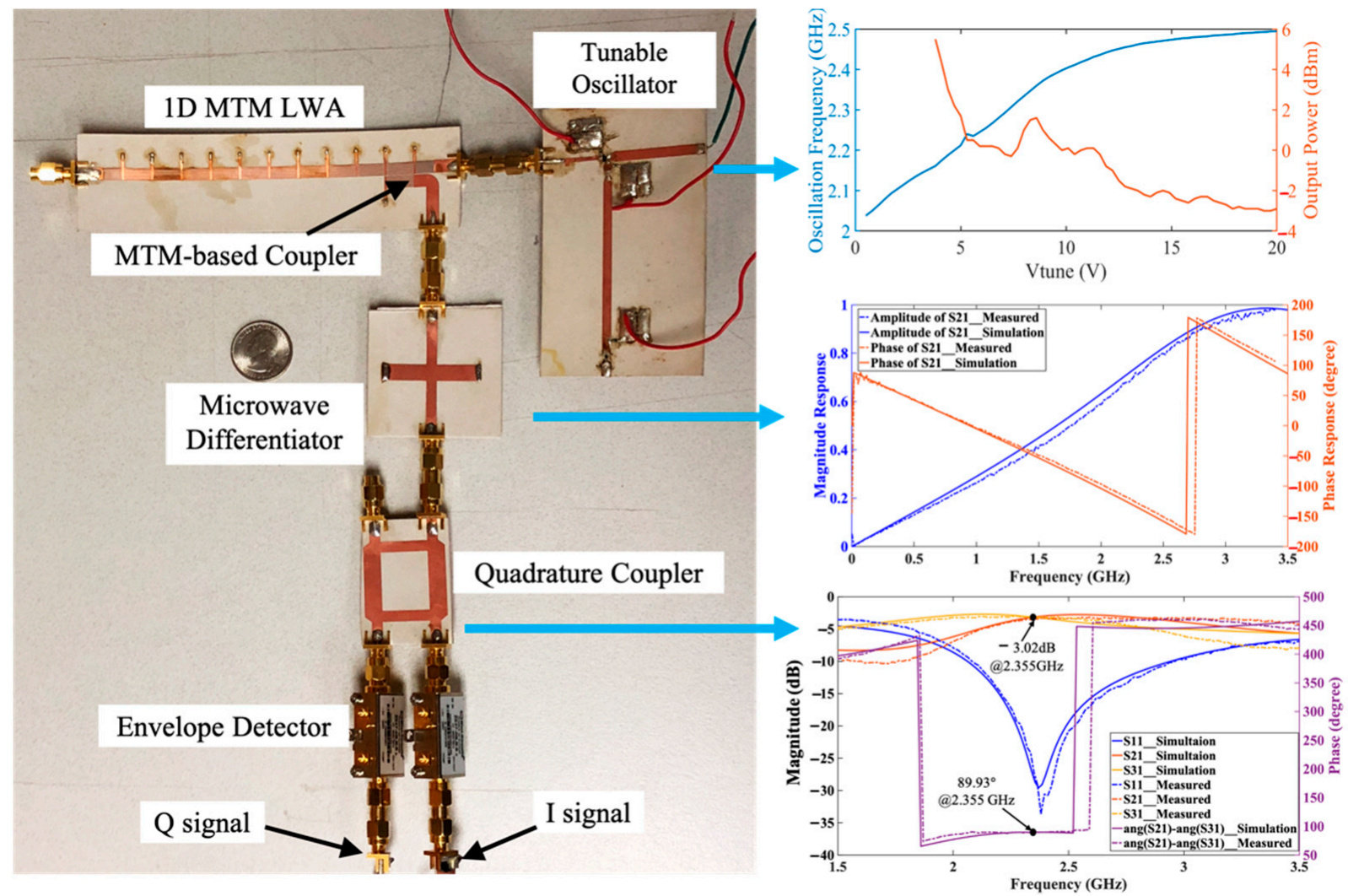

Figure 16. Prototype of the MTM LWA based on SIL radar sensor architecture at $2.4 \mathrm{GHz}$ band.

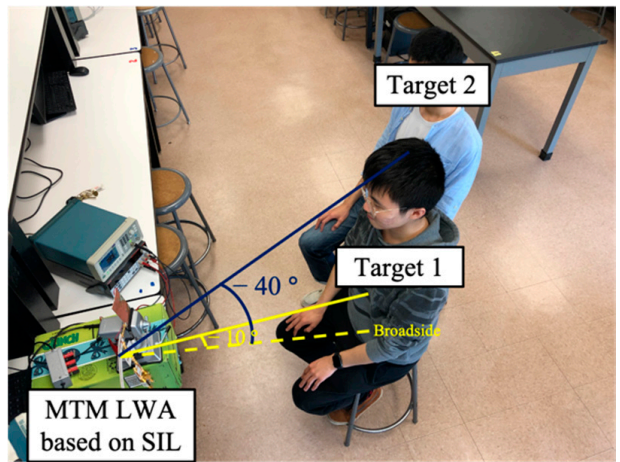

(a)

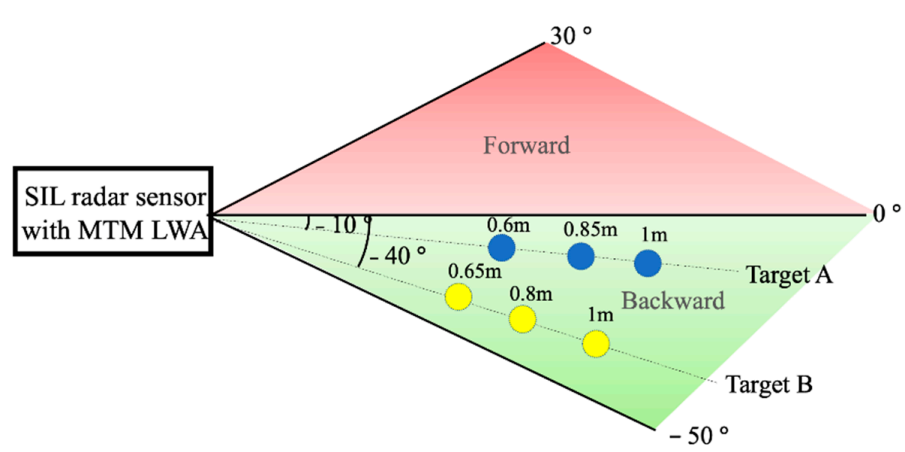

(b)

Figure 17. (a) Experimental setup for detecting vital sign signals of two targets at different locations using MTM LWA based on SIL radar sensor module; (b) its top view sketch [51].

The proposed MTM SIL radar sensor can track target location as well as detecting vital sign signals concurrently. For location tracking, the angular information of targets can be obtained from the dispersion relation based on the radiation pattern of MTM LWAs $[47,66]$. To further obtain the distance to the target, based on the frequency-shift keying (FSK) method $[67,68]$, two closely adjacent carrier frequencies $f_{1}$ and $f_{2}$ with a small frequency difference $\Delta f=f_{2}-f_{1}$ are chosen to illuminate the target, presented in Figure 19. In this case, the radiation angle $\theta$ for $f_{1}$ and $f_{2}$ are assumed to be the same, due to the small frequency difference. 

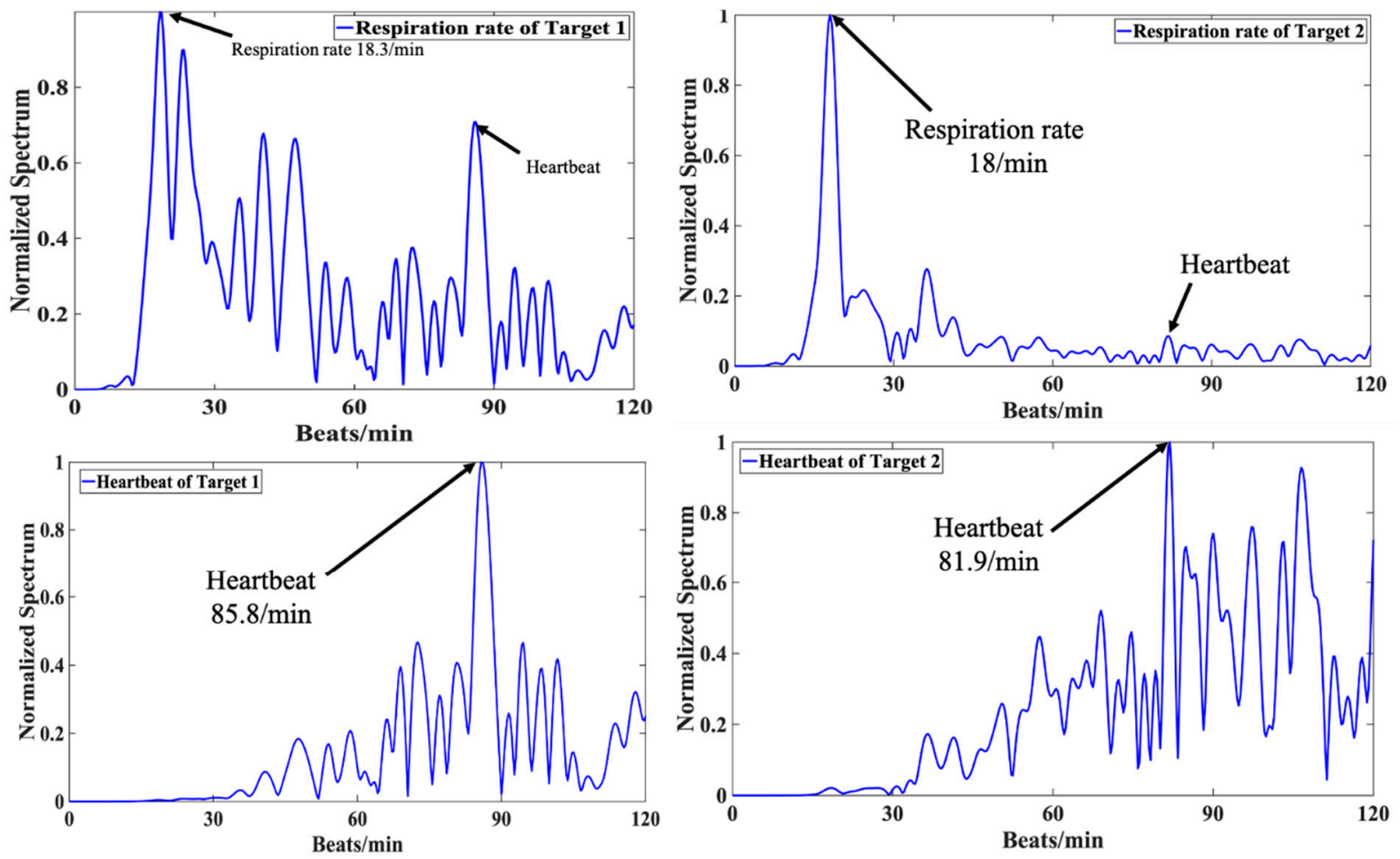

Figure 18. Measured respiration and heartbeat rates of Target 1 and 2 using MTM LWA based on SIL radar sensor module [51].

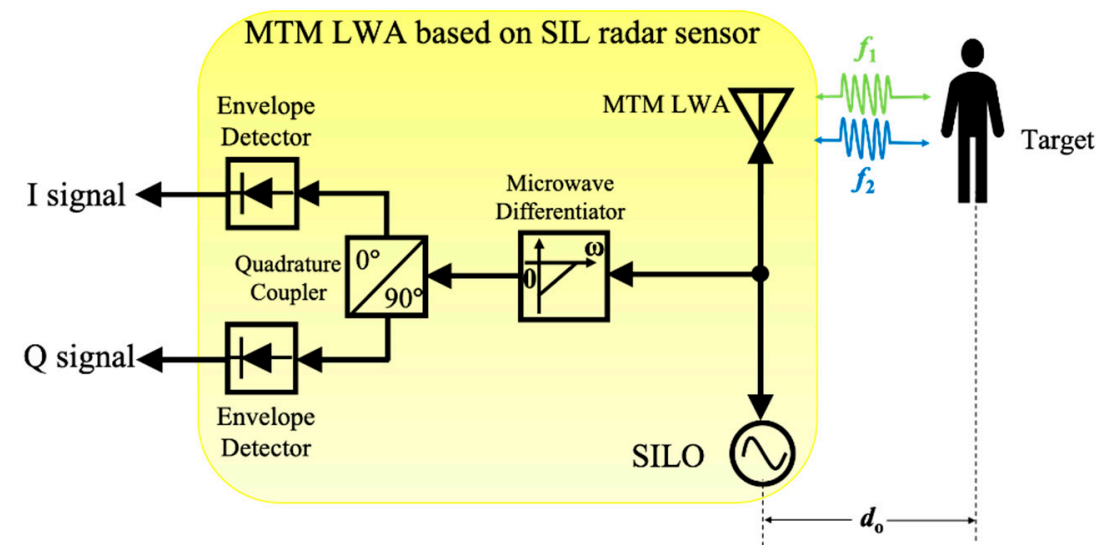

Figure 19. Frequency-shift keying (FSK) modulated SIL radar sensor for concurrent vital sign detection and location tracking of multiple targets.

To illustrate the method, the transmitted signal is represented as:

$$
T_{k}(t)=\cos \left[2 \pi f_{k} t+\Phi_{k}(t)\right],
$$

where $k=1,2$ and $\phi_{k}(t)$ are the phase noise from the voltage-controlled oscillator (VCO). When a human target is located at the distance of $d_{0}$, the reflected signal with phase including the distance information, is injected into the oscillator. Neglecting the amplitude response for simplicity, it can be expressed as

$$
R_{k}(t)=\cos \left[2 \pi f_{k} t-\frac{4 \pi\left(d_{0}+x_{v}(t)\right)}{\lambda_{k}}+\Phi_{k}\left(t-\frac{2 d_{0}}{c}\right)\right],
$$


where $x_{v}(t)$ represents the cardiac activity. According to Equation (19), because $x_{v}(t)$ is much smaller than the distance $d_{0}$, the phase term is approximately:

$$
\theta_{k}(t)=\frac{4 \pi\left(d_{0}+x_{v}(t)\right)}{\lambda_{k}} \approx \frac{4 \pi d_{0}}{\lambda_{k}},
$$

Therefore, by imposing the FSK method under pulse-width modulation (PWM) control, where the block diagram is shown in Figure 20, the phase difference of the two adjacent tones is:

$$
\Delta \theta=\theta_{2}-\theta_{1}=\frac{4 \pi d_{0}}{\lambda_{2}}-\frac{4 \pi d_{0}}{\lambda_{1}}=\frac{4 \pi \Delta f d_{0}}{c},
$$

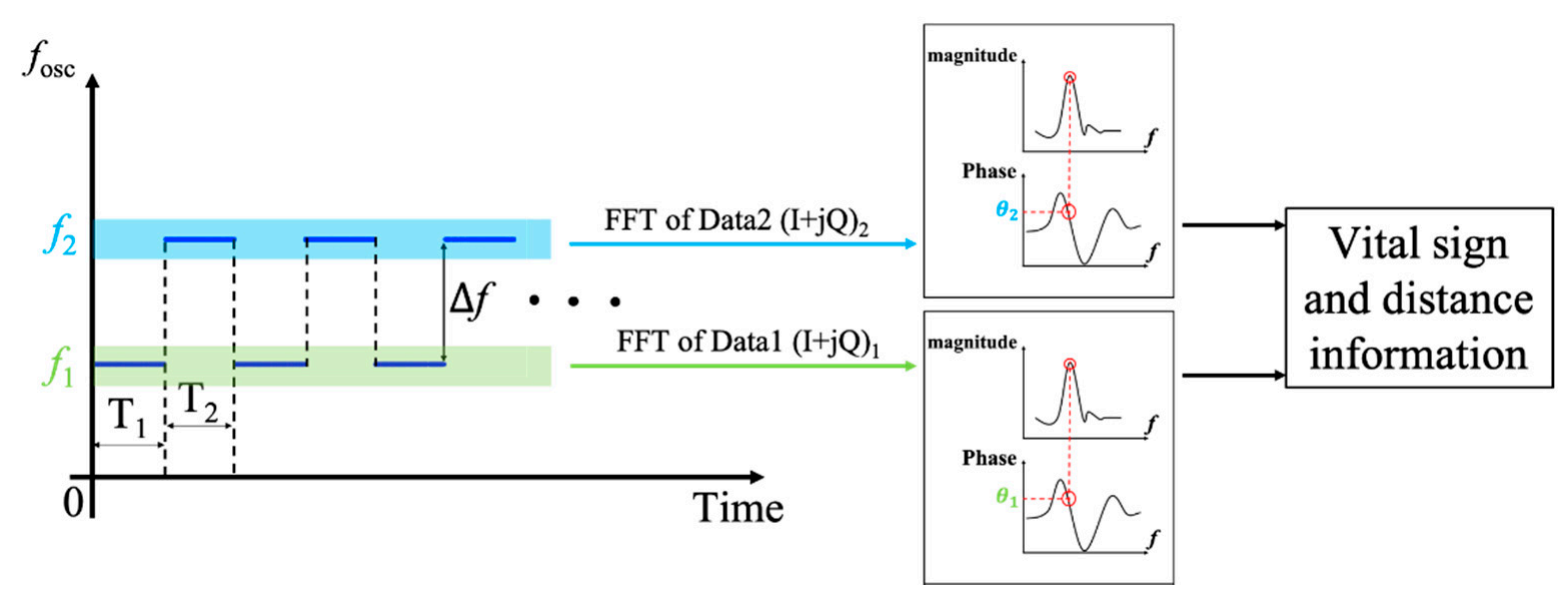

Figure 20. The block diagram of FSK signal processing under pulse-width modulation (PWM) control.

As such, the distance between the target and radar sensor can be calculated using:

$$
d_{0}=\frac{c \Delta \theta}{4 \pi \Delta f^{\prime}}
$$

If $f_{1}$ and $f_{2}$ are switched repeatedly at a small time interval of $T_{1}$ and $T_{2}$, respectively, $f_{1}$ and $f_{2}$ can be considered to work simultaneously. Meanwhile, because of the small frequency difference $\Delta f$, their frequency spectrums under the same Doppler frequency have almost the same amplitude response, but different phase responses. Consequently, once the phase difference $\Delta \theta$ is obtained through executing the FFT, the distance can be calculated from (23). Figure 21 depicts the I- and Q-baseband signals and their zoomed-in view in the time domain with the PWM control signal, when the target is at the distance of $0.65 \mathrm{~m}$. It is noted that the periodic amplitude change can be observed clearly, which is related to the chest motion due to the respiratory activity of the human target. The normalized spectrum and phase information of the target are presented in Figure 22.

The measured breath rate is 18 beats/min, which agrees well with the ground truth. Meanwhile, the phase response at the Doppler frequency, $f=0.3 \mathrm{~Hz}$, is utilized to calculate the distance through Equation (23), which gives $0.7 \mathrm{~m}$ with the actual distance of $0.65 \mathrm{~m}$. Along with the angular information obtained from the radiation pattern of the MTM LWA, the target location in 2D space can be obtained. In Figure 17b, when both targets sit at different locations in front of the radar sensor, the mean values of calculated distance with corresponding respiration and heartbeat rate are summarized in Tables 1 and 2, respectively. 


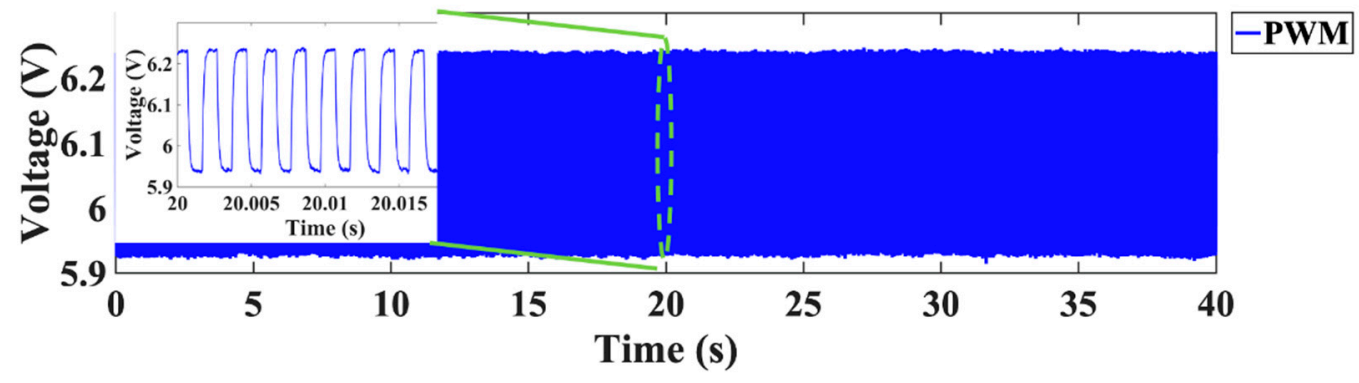

(a)

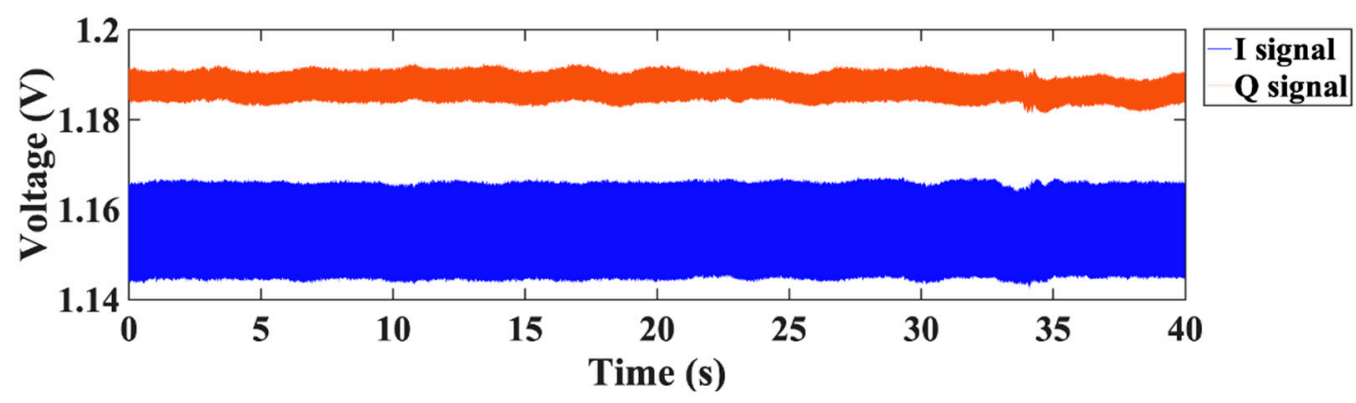

(b)
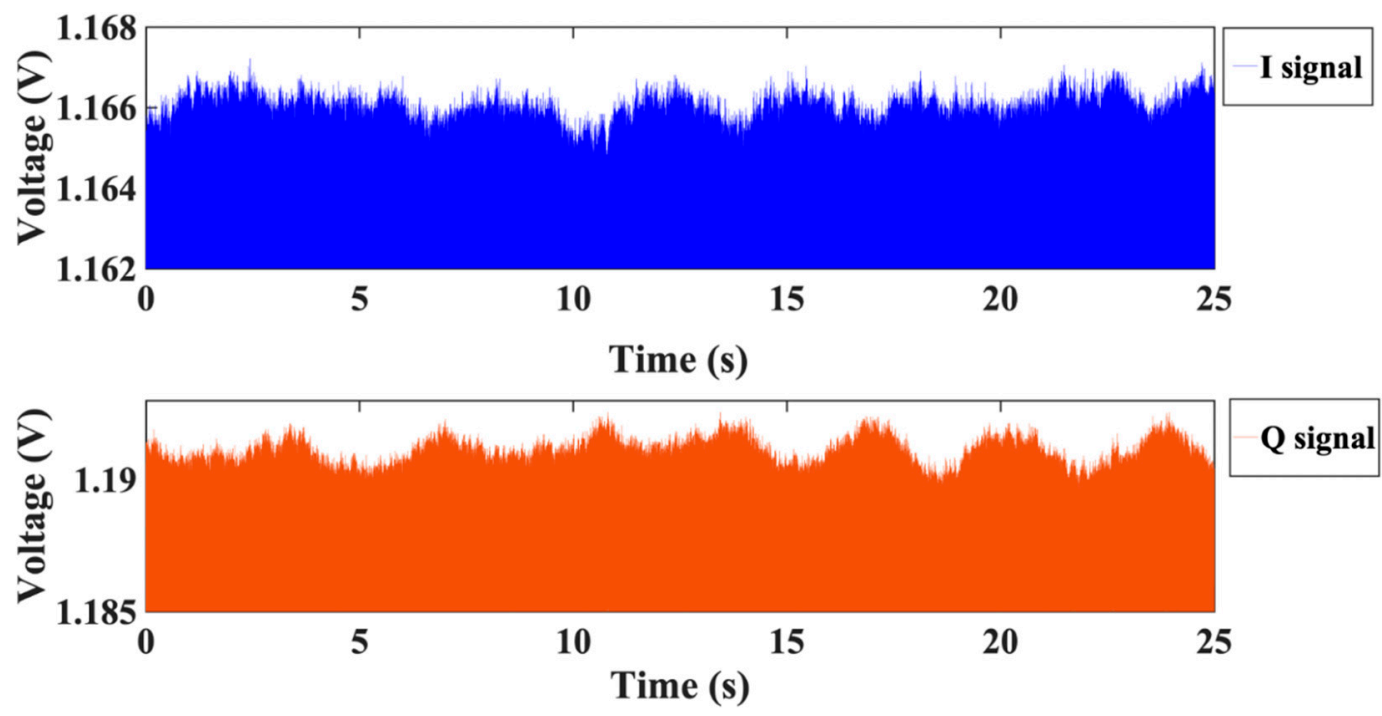

(c)

Figure 21. (a) Measured PWM control signal; (b) measured time domain I/Q signals and (c) their zoomed-in view of the target located at the distance of $0.65 \mathrm{~m} \mathrm{[51].}$ 

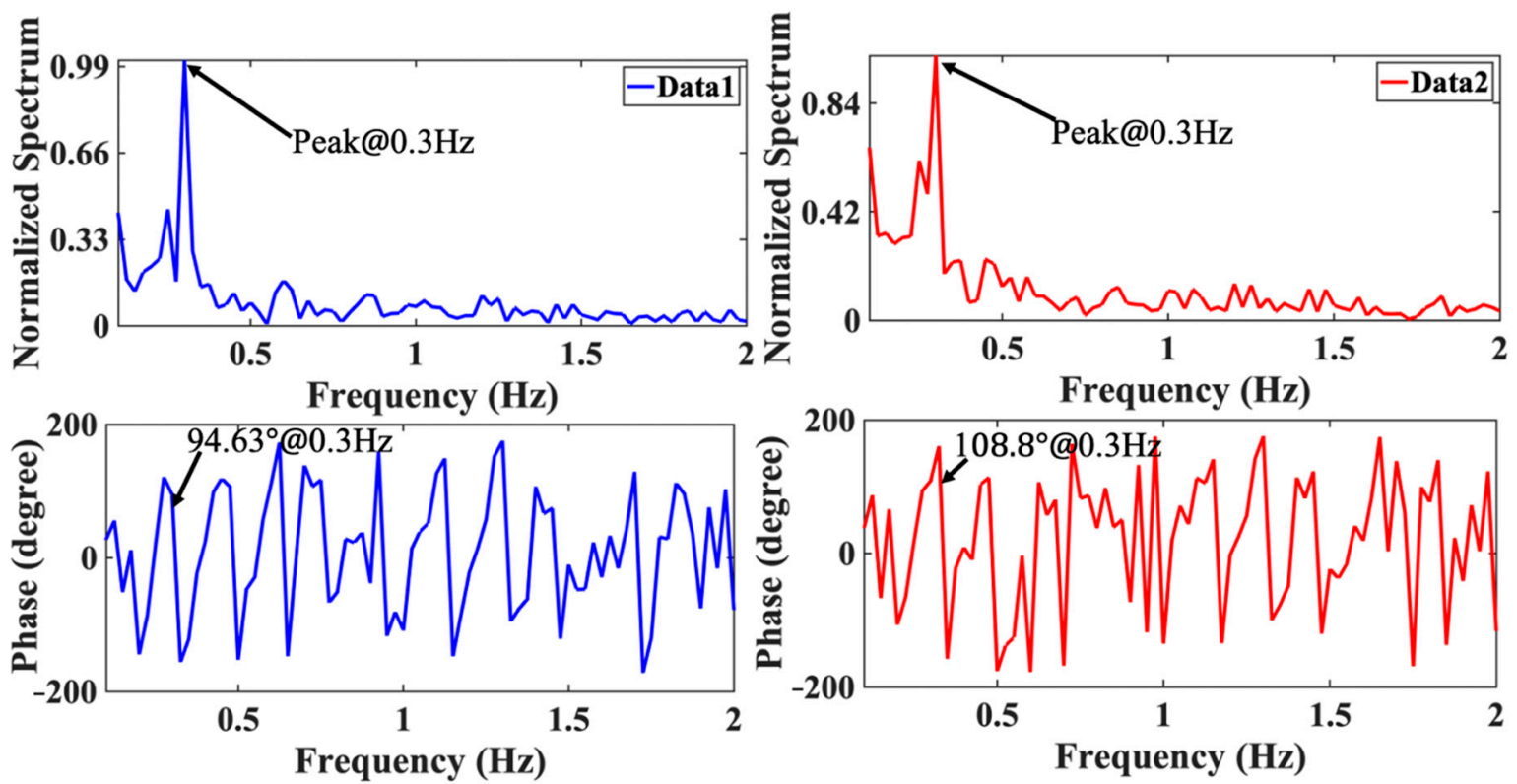

Figure 22. Normalized spectrum with phase information of the target located at the distance of $0.65 \mathrm{~m}$.

Table 1. Calculated distance results for both targets.

\begin{tabular}{ccccc}
\hline & $\begin{array}{c}\text { Mean of the Calculation } \\
\text { Results }(\mathbf{m})\end{array}$ & Ground Truth (m) & Error (m) & $\begin{array}{c}\text { Standard } \\
\text { Deviation }(\mathbf{m})\end{array}$ \\
\hline \multirow{3}{*}{ Target 1 } & 0.55 & 0.6 & 0.05 & 0.03 \\
& 0.81 & 0.85 & 0.04 & 0.04 \\
& 1.01 & 1 & -0.01 & 0.02 \\
\hline \multirow{3}{*}{ Target 2 } & 0.7 & 0.65 & 0.05 & 0.08 \\
& 0.77 & 0.8 & 0.03 & 0.06 \\
& 0.98 & 1 & 0.02 & 0.04 \\
\hline
\end{tabular}

${ }^{1}$ The uncertainty of the ground truth for distance is $0.08 \mathrm{~m}$.

Table 2. Vital sign results for both targets.

\begin{tabular}{cccccc}
\hline Distance (m) & $\begin{array}{c}\text { Respiration } \\
\text { (beats/min) }\end{array}$ & Ground Truth & $\begin{array}{c}\text { Heartbeat } \\
\text { (beats/min) }\end{array}$ & Ground Truth \\
\hline \multirow{3}{*}{ Target $\mathbf{1}$} & 0.6 & 18.3 & 18 & 85.8 & 87 \\
& 0.85 & 18 & 17 & 86.7 & 86 \\
\hline \multirow{3}{*}{ Target 2 } & 1 & 16.5 & 17 & 75 & 77 \\
& 0.65 & 18 & 18 & 81.9 & 81 \\
& 0.8 & 15 & 15 & 83.4 & 83 \\
& 1 & 15 & 16 & 79 & 80 \\
\hline
\end{tabular}

\section{Two-Dimensional (2D) Beam Scanning Metamaterial LWA Design}

As indicated in (5), the CRLH TL can be applied to fabricate the MTM LWA to achieve the frequency-dependent beam scanning capability. To realize the 2D beam scanning for vital sign detection, a 2D MTM LWA array operating at $24 \mathrm{GHz}$ band is proposed [52], which incorporates four 1D frequency-dependent beam scanning MTM LWAs with a series feeding network, shown in Figure 23. The series feeding network contributes to the different phase responses for four 1D frequency-dependent beam scanning MTM LWAs at one operation frequency, illustrated in Figure 24a. Therefore, 2D beam scanning can be realized by controlling the phase difference between each $1 \mathrm{D}$ antenna element. Figure $24 \mathrm{~b}$ shows the reflection coefficient $\left(S_{11}\right)$ is less than $-6 \mathrm{~dB}$ from 23.4 to $25.4 \mathrm{GHz}$. Within the same operation frequency range, its radiation pattern is shown in Figure 25. It can be seen that the main beam scans in a $2 \mathrm{D}$ fashion with respect to the frequency change. 


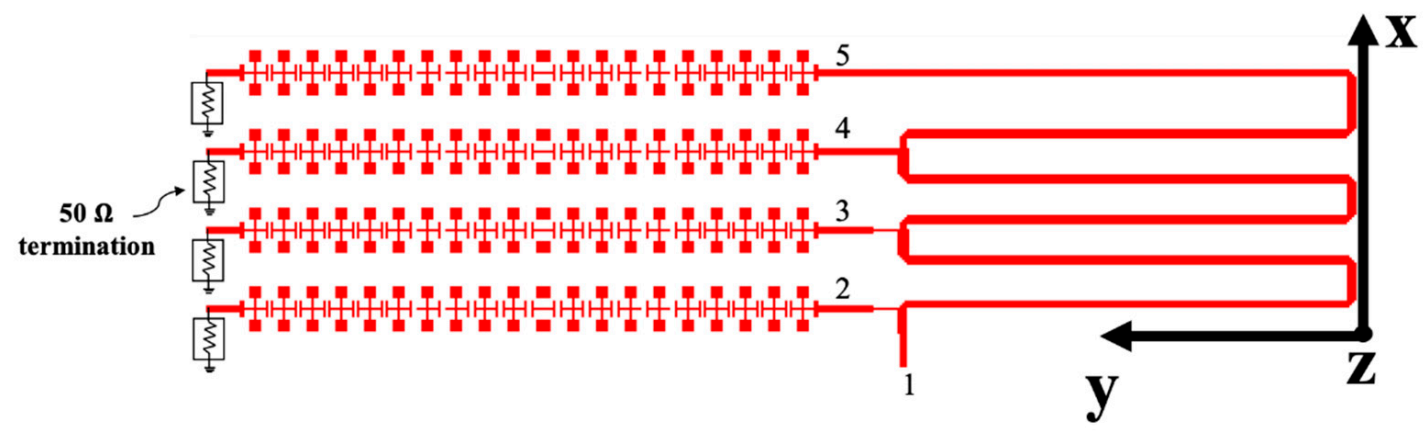

Figure 23. Layout of 2D MTM LWA array at $24 \mathrm{GHz}$ band [52].

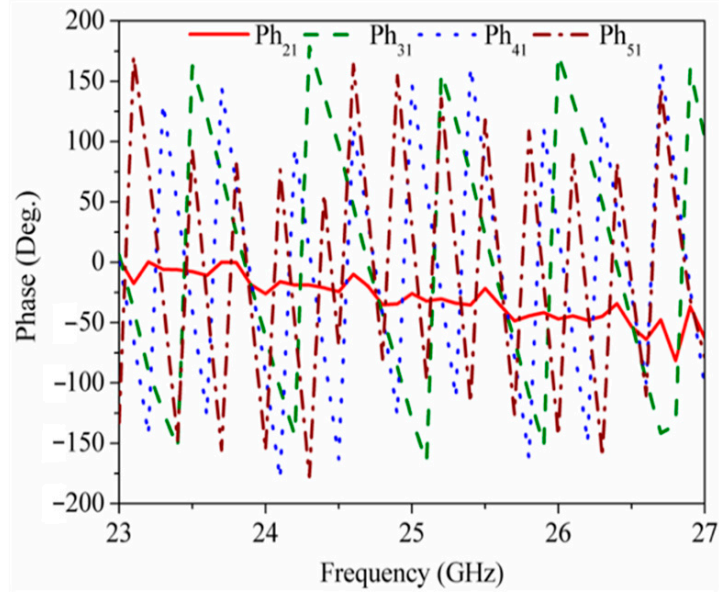

(a)

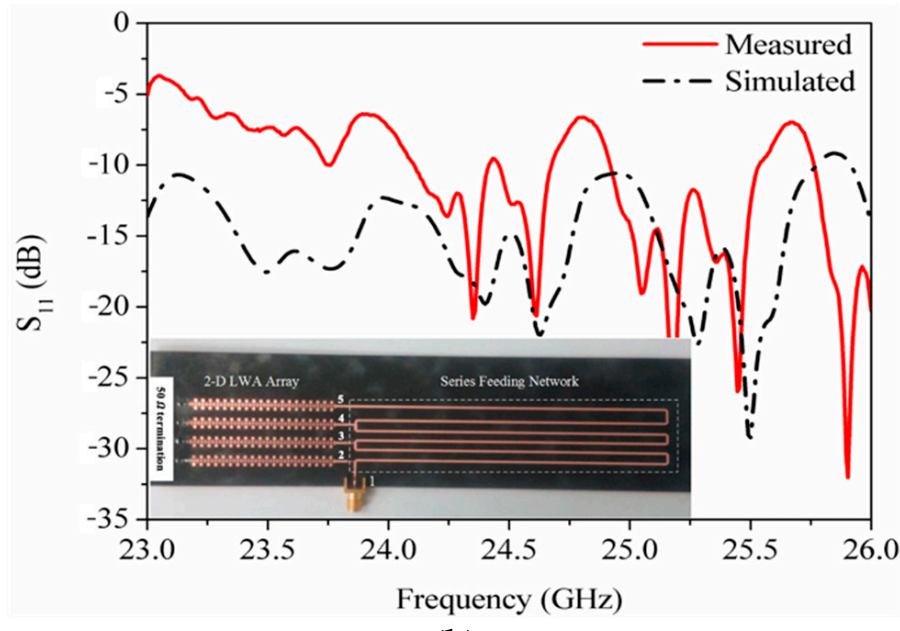

(b)

Figure 24. (a) Phase responses of the series feeding network; (b) S11 of 2D frequency-dependent beam scanning MTM LWA array [52].
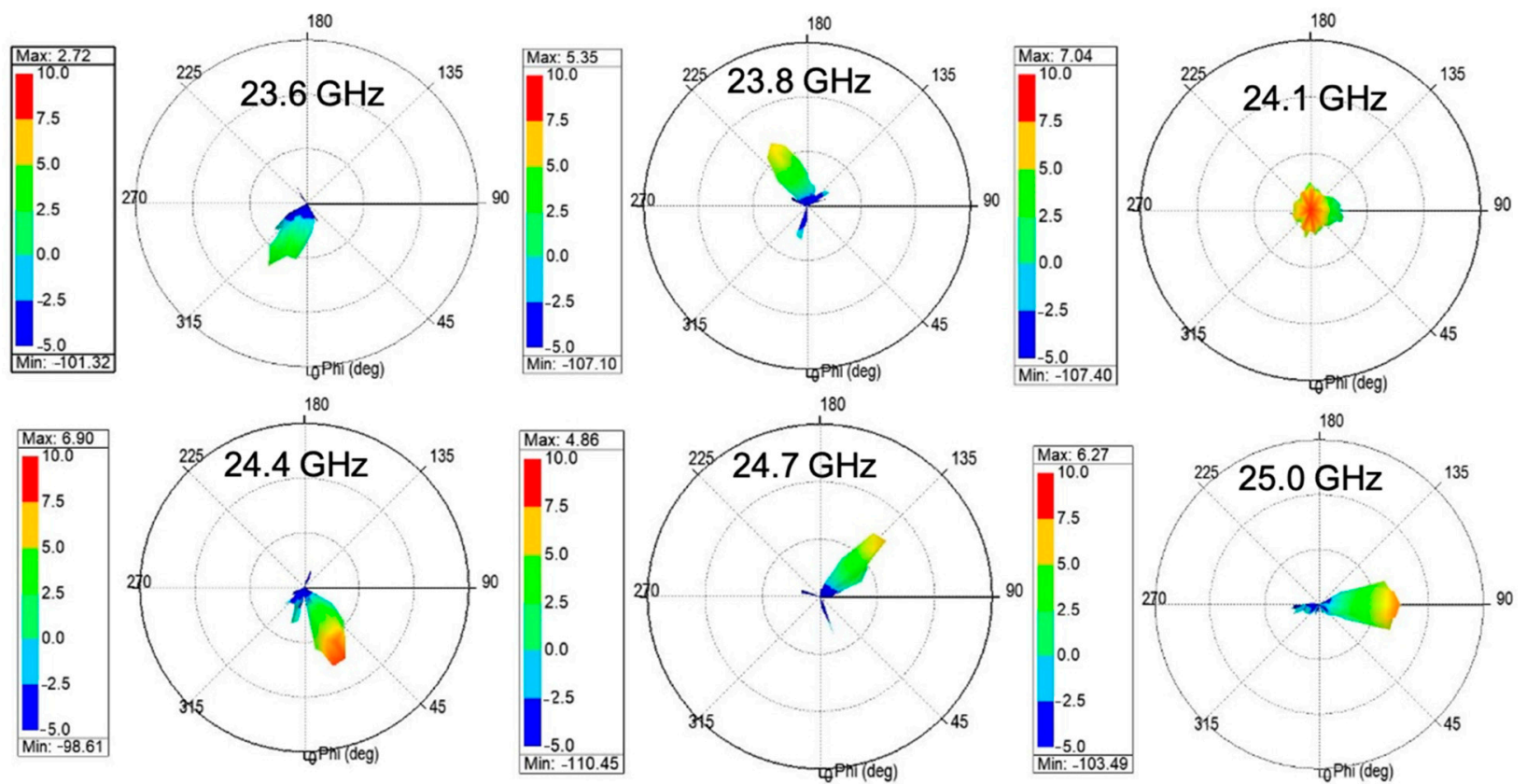

Figure 25. Radiation pattern of 2D MTM LWA array at $24 \mathrm{GHz}$ band [52]. 
As such, the designed 2D frequency-dependent beam scanning MTM LWA array can be used to detect the vital sign signals of multiple targets. To verify the 2D beam scanning capability, the designed MTM LWA array connected with a vector network analyzer (VNA) is utilized to detect vital sign signals of two persons with different heights, as shown in Figure 26. In this scenario, Person 1 sits with his chest facing the radiation angle of the main beam at $24.1 \mathrm{GHz}$, while Person 2 stands with his chest facing the radiation angle of the main beam at $24.4 \mathrm{GHz}$. By performing the FFT on the scattering parameters obtained from the VNA, vital sign signals, including respiration and heartbeat rates, can be obtained as shown in Figure 27. The measured respiration and heartbeat rates of Person 1 are 18 beats/min and 99 beats/min, respectively. Meanwhile, the measured respiration and heartbeat rates of Person 2 are 18 beats/min and 90 beats/min, respectively.

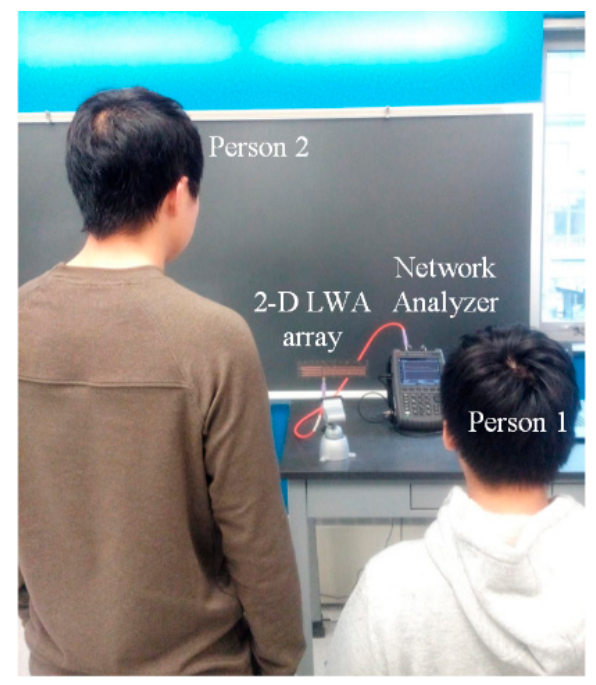

Figure 26. Experimental setup for multiple targets with different heights using 2D frequencydependent beam scanning MTM LWA array [52].
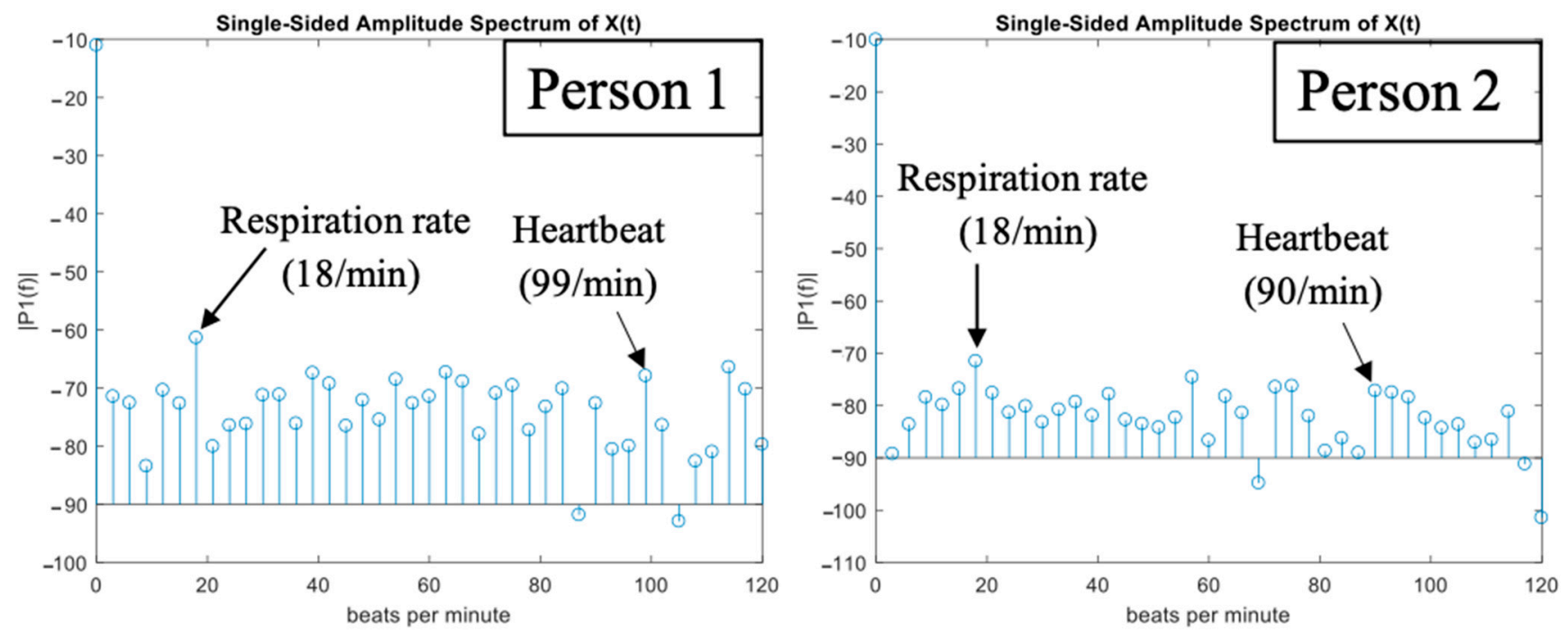

Figure 27. The measured respiration and heartbeat rates of two targets [52]. 


\section{Conclusions}

Over the past few decades, vital sign detection and location tracking using radar sensor modules have become a hot topic because of their non-contact and penetrable characteristics. With the development of semiconductors and algorithms, it has been demonstrated that different radar sensor architectures can obtain accurate vital sign information and target locations. On the other hand, multi-target detection based on radar sensor module is likely to be a trend for detecting vital sign information and location tracking. The main properties of different methods for multi-target detection using radar sensors are summarized in Table 3. As can be seen, MTM LWA-based vital radar sensors have several advantages such as low complexity and compact size. To this end, this paper reviews the recent development of radar sensors based on frequency-dependent beam scanning using MTM LWA, including MTM LWA-based homodyne architecture, MTM LWA using the self-injection locked (SIL) radar architecture, and 2D beam scanning MTM LWA design. Experimental detection results reveal the effectiveness of vital sign signals and location tracking for multiple targets using MTM LWAs based on these architectures.

Table 3. Main properties of methods for multi-target detection using radar sensors.

\begin{tabular}{cccc}
\hline Radar Configuration & Signal Processing Method & Number of Antennas Used & System Size \\
\hline Mechanical Rotors [23] & FFT & Low & Large \\
SIMO [29] & ADMS etc. & High & Medium \\
MIMO [26] & Frequency analysis etc. & High & Medium \\
RF switch-based radar system [30] & Heuristic digital signal processing & High & Medium \\
MTM LWA [51] & FFT & Low & Small \\
\hline
\end{tabular}

Author Contributions: C.-T.M.W. conceived and designed the experiments; Y.Y. performed the experiments analyzed the data and wrote the paper. All authors have read and agreed to the published version of the manuscript.

Funding: This research is funded by National Science Foundation (NSF) grant number ECCS-1818478.

Informed Consent Statement: Informed consent was obtained from all subjects involved in the study.

Data Availability Statement: The data presented in this study are available on request from the corresponding author.

Conflicts of Interest: The authors declare no conflict of interest.

\section{References}

1. Hu, W.; Zhao, Z.; Wang, Y.; Zhang, H.; Lin, F. Noncontact accurate measurement of cardiopulmonary activity using a compact quadrature doppler radar sensor. IEEE Trans. Biomed. Eng. 2014, 61, 725-735. [CrossRef] [PubMed]

2. Zhao, M.; Adib, F.; Katabi, D. Emotion recognition using wireless signals. In Proceedings of the 22nd Annual International Conference on Mobile Computing and Networking, New York, NY, USA, 3-7 October 2016; pp. 95-108.

3. Sakamoto, T.; Imasaka, R.; Taki, H.; Sato, T.; Yoshioka, M.; Inoue, K.; Fukuda, T.; Sakai, H. Feature-based correlation and topological similarity for interbeat interval estimation using ultrawideband radar. IEEE Trans. Biomed. Eng. 2016, 63, 747-757.

4. Nosrati, M.; Tavassolian, N. High-Accuracy Heart Rate Variability Monitoring Using Doppler Radar Based on Gaussian Pulse Train Modeling and FTPR Algorithm. IEEE Trans. Microw. Theory Tech. 2018, 66, 556-567. [CrossRef]

5. Muehlsteff, J.; Thijs, J.; Pinter, R.; Morren, G.; Muesch, G. A handheld device for simultaneous detection of electrical and mechanical cardiovascular activities with synchronized ECG and CW-Doppler Radar. In Proceedings of the EMBS, Lyon, France, 22-26 August 2007; pp. 5758-5761.

6. Buxi, D.; Redouté, J.M.; Yuce, M.R. Blood pressure estimation using pulse transit time from bioimpedance and continuous wave radar. IEEE Trans. Biomed. Eng. 2017, 64, 917-927. [CrossRef] [PubMed]

7. Thiel, F.; Kosch, O.; Seifert, F. Ultra-wideband sensors for improved magnetic resonance imaging, cardiovascular monitoring and tumour diagnostics. Sensors 2010, 10, 10778-10802. [CrossRef]

8. Lazaro, A.; David Girbau, D.; Villarino, R. Techniques for clutter suppression in the presence of body movements during the detection of respiratory activity through UWB radars. Sensors 2014, 14, 2595-2618. [CrossRef]

9. Lin, F.; Zhuang, Y.; Song, C.; Wang, A.; Li, Y.; Gu, C.; Li, C.; Xu, W. Sleepsense: A noncontact and cost-effective sleep monitoring system. IEEE Trans. Biomed. Circuits Syst. 2017, 11, 189-202. [CrossRef] 
10. Baboli, M.; Singh, A.; Soll, B.; Boric-Lubecke, O.; Lubecke, V.M. Good night: Sleep monitoring using a physiological radar monitoring system integrated with a polysomnography system. IEEE Microw. Mag. 2015, 16, 34-41. [CrossRef]

11. Vossiek, M.; Roskosch, R.; Heide, P. Precise 3-D Object position tracking using FMCW radar. In Proceedings of the 29th European Microwave Conference, Munich, Germany, 5-7 October 1999; pp. 234-237.

12. Wang, Y.; Liu, Q.; Fathy, A.E. Simultaneous localization and respiration detection of multiple people using low cost UWB biometric pulse Doppler radar sensor. In Proceedings of the IEEE MTT-S International Microwave Symposium (IMS), Montreal, QC, Canada, 17-22 June 2012; pp. 1-3.

13. Nguyen, V.; Pyun, J. Location detection and tracking of moving targets by a 2D IR-UWB radar system. Sensors 2015, 15, 6740-6762. [CrossRef]

14. Shingu, G.; Takizawa, K.; Ikegami, T. Human body detection using UWB radar in an indoor environment. In Proceedings of the International Symposium on Communications and Information Technologies, Vientiane, Laos, 21-23 October 2008; pp. 283-288.

15. Nishida, Y.; Murakami, S.; Hori, T.; Mizoguchi, H. Minimally privacy-violative human location sensor by ultrasonic radar embedded on ceiling. In Proceedings of the IEEE Sensors, Vienna, Austria, 24-27 October 2004; pp. $433-436$.

16. Yan, J.; Zhang, G.; Hong, H.; Chu, H.; Li, C.; Zhu, X. Phase-Based human target 2-D identification with a mobile FMCW radar platform. IEEE Trans. Microw. Theory Tech. 2019, 67, 5348-5359. [CrossRef]

17. Park, B.K.; Boric-Lubecke, O.; Lubecke, V.M. Arctangent demodulation with DC offset compensation in quadrature Doppler radar receiver systems. IEEE Trans. Microw. Theory Tech. 2007, 55, 1073-1079. [CrossRef]

18. Li, C.; Lin, J. Random body movement cancellation in Doppler radar vital sign detection. IEEE Trans. Microw. Theory Tech. 2008, $56,3143-3152$.

19. Mostafanezhad, I.; Boric-Lubecke, O.; Lubecke, V. A Coherent Low IF Receiver Architecture for Doppler Radar Motion Detector Used in Life Signs Monitoring. In Proceedings of the 2010 IEEE Radio and Wireless Symposium (RWS), New Orleans, LA, USA, 10-14 January 2010; pp. 571-574.

20. Nieh, C.-M.; Wei, C.; Lin, J. Concurrent Detection of Vibration and Distance Using Unmodulated CW Doppler Vibration Radar with an Adaptive Beam-Steering Antenna. IEEE Trans. Microw. Theory Tech. 2015, 63, 2069-2078. [CrossRef]

21. Mitomo, T.; Ono, N.; Hoshino, H.; Yoshihara, Y.; Watanabe, I.; Seto, I. A 77 GHz 90 nm CMOS transceiver for FMCW radar applications. IEEE J. Solid-State Circuits 2010, 45, 928-937. [CrossRef]

22. SeyyedEsfahlan, M.; Öztürk, E.; Kaynak, M.; Tekin, I. 77-GHz four element phased-array radar receiver front end. IEEE Trans. Compon. Packag. Manuf. Technol. 2016, 6, 1162-1173. [CrossRef]

23. Wang, G.; Gu, C.; Inoue, T.; Li, C. A Hybrid FMCW-Interferometry Radar for Indoor Precise Positioning and Versatile Life Activity Monitoring. IEEE Trans. Microw. Theory Tech. 2014, 62, 2812-2822. [CrossRef]

24. Nosrati, M.; Shahsavari, S.; Lee, S.; Wang, H.; Tavassolian, N. A Concurrent Dual-Beam Phased-Array Doppler Radar Using MIMO Beamforming Techniques for Short-Range Vital-Signs Monitoring. IEEE Trans. Antennas Propag. 2019, 67, 2390-2404. [CrossRef]

25. Lv, H.; Liu, M.; Jiao, T.; Zhang, Y.; Yu, X.; Li, S.; Jing, X.; Wang, J. Multi-target Human Sensing via UWB Bio-radar Based on Multiple Antennas. In Proceedings of the IEEE International Conference of IEEE Region 10 (TENCON 2013), Xian, China, 22-25 October 2013; pp. 1-4.

26. Cardillo, E.; Caddemi, A. A Review on Biomedical MIMO Radars for Vital Sign Detection and Human Localization. Electronics 2020, 9, 1497. [CrossRef]

27. Zhang, Z.; Nian, Y.; Chen, J.; He, M. An Experimental Study to Optimize the Stepped-Frequency Continuous-Wave Radar Parameters for Noncontact Multi-target Vital Sign Monitoring. In Proceedings of the 2019 IEEE International Conference on Computational Electromagnetics (ICCEM), Shangai, China, 20-22 March 2019; pp. 1-4.

28. Xiong, J.; Zhang, H.; Hong, H.; Zhao, H.; Zhu, X.; Li, C. Multi-target vital signs detection using SIMO continuous-wave radar with DBF technique. In Proceedings of the IEEE Radio and Wireless Symposium, San Antonio, TX, USA, 26-29 January 2020; pp. 194-196.

29. Wang, J.; Bai, X.; Gao, Q.; Li, X.; Bi, X.; Pan, M. Multi-Target Device-Free Wireless Sensing Based on Multiplexing Mechanisms. IEEE Trans. Veh. Tech. 2020, 69, 10242-10251. [CrossRef]

30. Fang, G.-W.; Huang, C.-Y.; Yang, C.-L. Switch-based low intermediate frequency system of a vital sign radar for simultaneous multitarget and multidirectional detection. IEEE J. Electromagn. RF Microw. Med. Biol. 2020, 4, 265-272. [CrossRef]

31. Lai, A.; Caloz, C.; Itoh, T. Composite right/left-handed transmission line metamaterials. IEEE Microw. Mag. 2004, 5, 34-50. [CrossRef]

32. Veselago, V.G. Electrodynamics of substances with simultaneously negative values of $\varepsilon$ and $\mu$. Usp. Fiz. Nauk 1967, 92, 517-548. [CrossRef]

33. Smith, D.R.; Padilla, W.J.; Vier, D.C.; Nemat-Nasser, S.C.; Schultz, S. Composite medium with simultaneously negative permeability and permittivity. Phys. Rev. Lett. 2000, 84, 4184. [CrossRef]

34. Caloz, C.; Okabe, H.; Iwai, H.; Itoh, T. Transmission line approach of left-handed materials. In Proceedings of the USNC/URSI Nat. Radio Science Meeting, San Antonio, TX, USA, 16-21 June 2002; pp. 39-42.

35. Iyer, A.K.; Eleftheriades, G.V. Negative refractive index metamaterials supporting 2-D waves. In Proceedings of the IEEE MTT-S International Microwave Symposium (IMS), Seattle, WA, USA, 2-7 June 2002; pp. 1067-1070.

36. Caloz, C.; Sanada, A.; Itoh, T. A novel composite right-/left-handed coupled-line directional coupler with arbitrary coupling level and broad bandwidth. IEEE Trans. Microw. Theory Tech. 2004, 52, 980-992. [CrossRef] 
37. Sanada, A.; Caloz, C.; Itoh, T. Characteristics of the composite right left-handed transmission lines. IEEE Microw. Wirel. Compon. Lett. 2004, 14, 68-70. [CrossRef]

38. Marqués, R.; Martin, F.; Sorolla, M. Metamaterials with Negative Parameters: Theory, Design, and Microwave Applications; John Wiley \& Sons: Hoboken, NJ, USA, 2008.

39. Caloz, C.; Itoh, T.; Rennings, A. CRLH metamaterial leaky-wave and resonant antennas. IEEE Antennas Propag. Mag. 2008, 50, 25-39. [CrossRef]

40. Paulotto, S.; Baccarelli, P.; Frezza, F.; Jackson, D.R. Full-Wave modal dispersion analysis and broadside optimization for a class of microstrip CRLH leaky-wave antennas. IEEE Trans. Microw. Theory Tech. 2008, 56, 2826-2837. [CrossRef]

41. Kawaguchi, T.; Deguchi, H.; Tsuji, M. A Unit Cell Construction Based on Equivalent-Circuit Elements for Microstrip LeakyWave Antennas. In Proceedings of the 2019 International Conference on Electromagnetics in Advanced Applications (ICEAA), Granada, Spain, 9-13 September 2019; pp. 0641-0645.

42. Javanbakht, N.; Syrett, B.; Amaya, R.E.; Shaker, J. Electric beam-steering metamaterial leaky-wave antenna. In Proceedings of the 2019 International Conference on Electromagnetics in Advanced Applications (ICEAA), Granada, Spain, 9-13 September 2019; pp. 0325-0327.

43. Oliner, A.A. Radiating Periodic Structures: Analysis in Terms of $k$ vs. $\beta$ Diagrams, Short Course on Microwave Field and Network Techniques; Polytechnic Institute of Brooklyn: New York, NY, USA, 1963.

44. Crepeau, P.J.; Mclsaac, P.R. Consequencies of symmetry in periodic structures. In Proceedings of the IEEE; IEEE: New York, NY, USA, 1964; pp. 33-43.

45. Zhang, G.; Zhang, Q.; Chen, Y.; Murch, R.D. High-scanning-rate and wide-angle leaky-wave antennas based on glide-symmetry Goubau line. IEEE Trans. Antennas Propag. 2020, 68, 2531-2540. [CrossRef]

46. Jang, T.; Choi, J.; Lim, S. Compact coplanar waveguide (CPW)-fed zeroth-order resonant antennas with extended bandwidth and high efficiency on vialess single layer. IEEE Trans. Antennas Propag. 2011, 59, 363-372. [CrossRef]

47. Wu, C.-T.M. A real-time multiple target detecting scheme based on microwave metamaterials. In Proceedings of the 2015 European Microwave Conference (EuMC), Paris, France, 6-11 September 2015; pp. 497-500.

48. Salarkaleji, M.; Ali, M.A.; Wu, C.-T.M. Two-dimensional full hemisphere frequency scanning array based on metamaterial leaky wave antennas and feed networks. In Proceedings of the IEEE MTT-S International Microwave Symposium (IMS), San Francisco, CA, USA, 22-27 May 2016; pp. 1-4.

49. Lu, C.; Yuan, Y.; Tseng, C.-H.; Wu, C.-T.M. Multi-Target Continuous-Wave Vital Sign Radar using 24 GHz Metamaterial Leaky Wave Antennas. In Proceedings of the IEEE MTT-S International Microwave Biomedical Conference (IMBioC), Nanjing, China, 6-8 May 2019; pp. 1-4.

50. Lu, C.; Yuan, Y.; Tseng, C.-H.; Wu, C.-T.M. Multi-target motion detection radar sensor using 24GHz metamaterial leaky wave antennas. In Proceedings of the IEEE MTT-S International Conference on Microwaves for Intelligent Mobility (ICMIM), Detroit, MI, USA, 15-16 April 2019; pp. 1-3.

51. Yuan, Y.; Lu, C.; Chen, A.Y.-K.; Tseng, C.-H.; Wu, C.-T.M. Multi-target concurrent vital sign and location detection using metamaterial-integrated self-injection-locked quadrature radar sensor. IEEE Trans. Microw. Theory Tech. 2019, 67, 5429-5437. [CrossRef]

52. Li, Q.; Zhang, Y.; Wu, C.-T.M. Noncontact Vital Sign Detection using 24GHz Two-Dimensional Frequency Scanning Metamaterial Leaky Wave Antenna Array. In Proceedings of the IEEE MTT-S International Microwave Symposium (IMS), Philadelphia, PA, USA, 10-15 June 2018; pp. 255-258.

53. Yang, Z.-k.; Zhao, S.; Huang, X.-D.; Lu, W. Accurate Doppler radar-based heart rate measurement using matched filter. IEICE Electron. Expr. Lett. 2020, 17, 1-6. [CrossRef]

54. Rahman, A.; Yavari, E.; Gao, X.; Lubecke, V.; Boric-Lubecke, O. Signal processing techniques for vital sign monitoring using mobile short range Doppler radar. In Proceedings of the IEEE Topical Conference on Biomedical Wireless Technologies, Networks, and Sensing Systems (BioWireleSS), San Diego, CA, USA, 25-28 January 2015; pp. 17-19.

55. Wang, J.; Wang, X.; Chen, L.; Huangfu, J.; Li, C.; Ran, L. Non-contact Distance and Amplitude Independent Vibration Measurement Based on an Extended DACM Algorithm. IEEE Trans. Instrum. Meas. 2014, 63, 145-153. [CrossRef]

56. Rittiplang, A.; Phasukkit, P. UWB Radar for Multiple Human Detection through the Wall Based on Doppler Frequency and Variance Statistic. In Proceedings of the 2019 12th Biomedical Engineering International Conference (BMEiCON), Ubon Ratchathani, Thailand, 19-22 November 2019; pp. 1-5.

57. Walterscheid, I.; Biallawons, O.; Berens, P. Contactless Respiration and Heartbeat Monitoring of Multiple People Using a 2-D Imaging Radar. In Proceedings of the 2019 41st Annual International Conference of the IEEE Engineering in Medicine and Biology Society (EMBC), Berlin, Germany, 23-27 July 2019; pp. 3720-3725.

58. Otto, S.; Rennings, A.; Solbach, K.; Caloz, C. Transmission line modeling and asymptotic formulas for periodic leaky-wave antennas scanning through broadside. IEEE Trans. Antennas Propag. 2011, 59, 3695-3709. [CrossRef]

59. Gomez-Diaz, J.S.; Canete-Rebenaque, D.; Alvarez-Melcon, A. A simple CRLH LWA circuit condition for constant radiation rate. IEEE Antennas Wirel. Propag. Lett. 2011, 10, 29-32. [CrossRef]

60. Droitcour, A.D.; Boric-Lubecke, O.; Lubecke, V.M.; Lin, J.; Kovacs, G.T.A. Range correlation and I/Q performance benefits in single-chip silicon Doppler radars for noncontact cardiopulmonary monitoring. IEEE Trans. Microw. Theory Tech. 2004, 52, 838-848. [CrossRef] 
61. Wang, F.K.; Li, C.J.; Hsiao, C.H.; Horng, T.S.; Lin, J.; Peng, K.C.; Jau, J.K.; Li, J.Y.; Chen, C.C. A novel vital-sign sensor based on a self-injection-locked oscillator. IEEE Trans. Microw. Theory Tech. 2010, 58, 4112-4120. [CrossRef]

62. Wang, F.-K.; Horng, T.-S.; Peng, K.-C.; Jau, J.-K.; Li, J.-Y.; Chen, C.-C. Single-antenna Doppler radars using self and mutual injection locking for vital sign detection with random body movement cancellation. IEEE Trans. Microw. Theory Tech. 2011, 59, 3577-3587. [CrossRef]

63. Gonzalez, G. Foundations of Oscillator Circuit Design; Artech House: Boston, MA, USA, 2007.

64. Tseng, C.-H.; Yu, L.-T.; Huang, J.-K.; Chang, C.-L. A wearable self-injection-locked sensor with active integrated antenna and differentiator-based envelope detector for vital-sign detection from chest wall and wrist. IEEE Trans. Microw. Theory Tech. 2018, 66, 2511-2521. [CrossRef]

65. Hsue, C.-W.; Tsai, L.-C.; Chen, K.-L. Implementation of first-order and second-order microwave differentiators. IEEE Trans. Microw. Theory Tech. 2004, 52, 1443-1448. [CrossRef]

66. Wu, C.-T.M.; Sun, J.S. Microwave metamaterials-based ultrafast detecting scheme for automotive radars. Microw. Opt. Technol. Lett. 2015, 57, 1343-1345. [CrossRef]

67. Wang, J.; Li, C. A human tracking and physiological monitoring fsk technology for single senior at home care. In Proceedings of the 2018 40th Annual International Conference of the IEEE Engineering in Medicine and Biology Society (EMBC), Honolulu, HI, USA, 18-21 July 2018; pp. 4432-4435.

68. Rohling, H.; Moller, C. Radar waveform for automotive radar systems and applications. In Proceedings of the IEEE Radar Conference, Rome, Italy, 26-30 May 2008; pp. 1-3. 